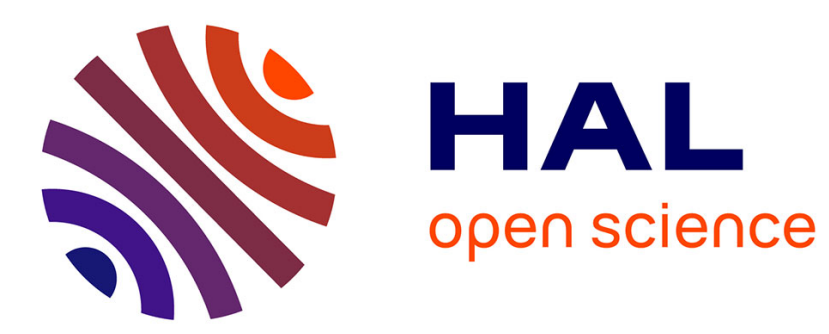

\title{
Performance assessment of a new class of local absorbing boundary conditions for elliptical- and prolate spheroidal-shaped boundaries
}

\author{
Hélène Barucq, Rabia Djellouli, Anne-Gaëlle Saint-Guirons
}

\section{To cite this version:}

Hélène Barucq, Rabia Djellouli, Anne-Gaëlle Saint-Guirons. Performance assessment of a new class of local absorbing boundary conditions for elliptical- and prolate spheroidal-shaped boundaries. Applied Numerical Mathematics, 2009, 59 (7), pp.1467-1498. 10.1016/j.apnum.2008.10.001 。 inria-00338494

\section{HAL Id: inria-00338494 \\ https://hal.inria.fr/inria-00338494}

Submitted on 13 Nov 2008

HAL is a multi-disciplinary open access archive for the deposit and dissemination of scientific research documents, whether they are published or not. The documents may come from teaching and research institutions in France or abroad, or from public or private research centers.
L'archive ouverte pluridisciplinaire $\mathbf{H A L}$, est destinée au dépôt et à la diffusion de documents scientifiques de niveau recherche, publiés ou non, émanant des établissements d'enseignement et de recherche français ou étrangers, des laboratoires publics ou privés. 


\title{
Performance assessment of a new class of local absorbing boundary conditions for elliptical- and prolate spheroidal-shaped boundaries
}

\author{
H. Barucq ${ }^{1,2}$, R.Djellouli ${ }^{3,1}$, A. Saint-Guirons ${ }^{2,1}$, \\ ${ }^{1}$ INRIA Bordeaux Sud-Ouest Research Center Team-Project Magique3D \\ ${ }^{2}$ Laboratoire de Mathématiques Appliquées, CNRS UMR 5142, Université de Pau \\ et des Pays de l'Adour, IPRA-Avenue de l'Université, 64013 Pau, France \\ ${ }^{3}$ Department of Mathematics, California State University Northridge, CA \\ 91330-8313, USA
}

\begin{abstract}
New approximate local DtN boundary conditions are proposed to be applied on elliptical- or prolate-spheroid exterior boundaries when solving respectively twoor three-dimensional acoustic scattering problems by elongated obstacles. These new absorbing conditions are designed to be exact for the first modes. They can be easily incorporated in any finite element parallel code while preserving the local structure of the algebraic system. Unlike the standard approximate local DtN boundary conditions that are restricted to circular- or spherical-shaped boundaries, the proposed conditions are applicable to exterior elliptical-shaped boundaries that are more suitable for surrounding elongated scatterers because they yield to smaller computational domains. The mathematical and numerical analysis of the effect of the frequency and the eccentricity values of the boundary on the accuracy of these conditions, when applied for solving radiating and scattering problems, reveals in particular- that the new second-order DtN boundary condition retains a good level of accuracy, in the low frequency regime, regardless of the slenderness of the boundary.
\end{abstract}

Key words: Absorbing boundary conditions, elliptic coordinates, prolate spheroidal coordinates, Dirichlet-to-Neumann operator, Helmholtz equation, radiating problems, scattering problems.

Email address: helene.barucq@univ-pau.fr, rabia.djellouli@csun.edu, anne-gaelle.saint-guirons@univ-pau.fr (H. Barucq ${ }^{1,2}$, R.Djellouli $^{3,1}$, A. Saint-Guirons $\left.{ }^{2,1}\right)$.

Preprint submitted to Applied Numerical Mathematics $\quad 15$ October 2008 


\section{Introduction}

The computation of the solutions of exterior Helmholtz problems requires first to reformulate them in a finite domain. This is often achieved by surrounding the given radiator or scatterer(s) by an artificial boundary that is located at some distance, measured in multiples of wavelength of interest, from its surface. A so-called "nonreflecting" boundary condition is then prescribed on the artificial boundary to represent the "far-field" behavior of the scattered field. The challenge here is the development of a simple but reliable as well as cost-effective computational procedure for representing the far-field behavior of the scattered field. Hence, the efficiency of such boundary conditions affects significantly the accuracy, the computational complexity, as well as the costeffectiveness of any solution methodology for this class of wave problems that are very important to many applications such as sonar, radar, geophysical exploration, nondestructive testing, etc. Given that, various absorbing boundary conditions have been designed for over seventy years, and the quest for such conditions is still ongoing (see, e.g., the recent review by Turkel in [29] and the paper of $[22])$.

This work is devoted to the construction and the analysis of a new class of approximate local Dirichlet-to-Neumann (DtN) boundary conditions to be employed on elliptical or prolate-spheroid boundaries that are primary candidates for surrounding elongated scatterers such as submarines. The idea for constructing such conditions is mainly motivated by the following three reasons. First, the widely-used second-order absorbing boundary conditions (BGT2) designed by Bayliss, Gunzbürger and Turkel for circular- and spherical-shaped boundaries [6] performs poorly in the low frequency regime when they are expressed in elliptical coordinates and applied to elliptical-shaped or prolatespheroid boundaries. Their accuracy deteriorates significantly for large eccentricity values of the boundaries as reported in [24]. The damping effect introduced to this class of conditions [25] improves the performance for only small eccentricity values. However, the modified BGT2 conditions still perform poorly for eccentricity values larger than 0.6 in the (relatively) low frequency regime (see Figures 6 and 15 in [25]). Hence, there is a need for constructing local absorbing boundary conditions (ABC) that extend the range of satisfactory performance. Second, the two-dimensional approximate local DtN condition designed for circular-shaped boundaries [13] outperforms significantly BGT condition, particularly for low wavenumber values as reported in [18]. Third, the three-dimensional approximate local DtN condition designed for spherical-shaped boundaries [17], which is identical to BGT2 conditions, perform very well for low wavenumber values as reported in [18]. However, using these approximate local DtN conditions on circular-shaped (2D) or on spherical-shaped (3D) exterior boundaries when solving scattering problems by elongated scatterer often leads to larger than needed computational do- 
mains, which hampers the computational efficiency of the proposed solution methodology. This suggests that approximate local DtN boundary conditions designed for elliptical-shaped boundaries (2D) or prolate-spheroid boundaries (3D) is an attractive alternative for improving the computational performance.

We propose in this paper to construct such absorbing boundary conditions and to assess analytically and numerically their performance when employed for solving two- and three-dimensional exterior Helmholtz problems.

The idea for constructing approximate local DtN boundary conditions is not new. Indeed, as stated earlier, such conditions have been already derived for circular-shaped [13] and spherical-shaped boundaries [17]. The construction procedure adopted in [13] and [17] is based on the localization of the truncated global DtN boundary condition [20]. The key ingredient of this procedure is the trigonometric identities that express high order derivatives of sine and cosine functions (see, e.g., Eq. (A4) p. 276 in [13]). However, these properties are not satisfied neither by the periodic Mathieu functions [28] nor by the angular spheroidal wave functions [10]. Consequently, the procedure used in $[13,17]$ is no longer applicable to the truncated global DtN boundary operator when expressed in elliptical or prolate spheroidal coordinates $[15,14]$. Our approach for constructing the class of approximate local DtN boundary conditions in elliptical coordinates (2D) and in prolate spheroidal coordinates (3D) can be viewed as an inverse-type approach. More specifically, we first consider Robintype boundary conditions whose coefficients are unknown. However, unlike the case of polar or spherical coordinates, the coefficients of these conditions depend on the angle $\theta$ of the elliptical coordinates in $2 \mathrm{D}$, and on the angles $(\varphi, \theta)$ of the prolate spheroidal coordinates in 3D. Such dependence is necessary for preserving both the symmetry and local nature of the resulting boundary conditions. Second, we require these conditions to be exact representations of the first modes. Consequently, the coefficients are determined by solving a linear algebraic system.

We investigate mathematically and numerically the effect of low wavenumber and eccentricity on the performance of the constructed approximate local DtN boundary conditions. This study is conducted in the context of the so-called on-surface radiation condition formulation (OSRC) [21]. Such formulation allows to perform the investigation analytically. However, the OSRC approach is not appropriate for high frequency regime especially when the creeping rays are not correctly modeled, as previously observed in [2]. Since then, many attempts have been made to include these complex contributions in the high frequency regime $[3,8,9,19]$. We must emphasis that he main interest in the following analyses is to evaluate the performance of the proposed approximate local DtN conditions at low wavenumber to find out -in particular- if relatively small computational domains can be employed in order to avoid excessive computational cost. The OSRC formulation must be viewed as an extreme case 
while an artificial elliptical-shaped or prolate-spheroid boundary surrounding an elongated scatterer at a fraction of wavelength would be less "demanding" on the considered absorbing boundary condition. The proposed study reveals that the constructed second-order local DtN conditions, when applied for solving low frequency acoustic scattering problems, not only outperforms BGT2 conditions but more importantly extends the range of satisfactory performance to all eccentricity values of the elliptical-shaped and prolate-spheroid boundaries. This result demonstrates that the proposed second-order DtN boundary condition is very efficient for solving scattering problems by very elongated obstacles.

The reminder of this paper is as follows. In Section 2, we specify the nomenclature and assumptions, introduce the new two-dimensional approximate local DtN boundary conditions, and then assess analytically and numerically their performance when applied for (a) computing single elliptical modes, and (b) solving two-dimensional acoustic scattering problems. In Section 3, we study the three-dimensional case. We state our conclusions in Section 4.

\section{Two-dimensional exterior Helmholtz problems}

We introduce in this section the two-dimensional approximate local DtN boundary conditions and assess their performance when applied for solving low frequency radiating and scattering problems. More specifically, we investigate analytically and numerically the effect of low wavenumber and eccentricity on the performance of the constructed approximate local DtN boundary conditions in the context of the OSRC formulation.

\subsection{Preliminaries}

Throughout this section, we use the elliptical coordinates $(\xi, \theta)$ which are related to the rectangular cartesian ones $(x, y)$ by $x=a \cos \theta, y=b \sin \theta$, where $\theta \in[0,2 \pi)$. The parameters $a$ and $b$ represent respectively the semimajor and semi-minor axes of the ellipse and are given by $a=f \cosh \xi$ and $b=f \sinh \xi ; \xi$ is a strictly positive real number and $f=\sqrt{a^{2}-b^{2}}$ is the interfocal distance. Finally, we define the eccentricity $e$ on the ellipse $\xi=\xi_{0}$ by:

$$
e=\frac{1}{\cosh \xi_{0}}=\sqrt{1-\frac{b^{2}}{a^{2}}}
$$

Note that the eccentricity $e$ characterizes the slenderness of the boundary. It satisfies $0<e<1$. When $e \rightarrow 0$, the ellipse becomes a circle, and the ellipse degenerates into a line segment with length $2 f$ as $e \rightarrow 1$. 
We recall that the $n^{\text {th }}$ elliptical mode $u_{n}$ is given by [28] :

$$
u_{n}= \begin{cases}\operatorname{Re}_{n}^{(3)}(k f, \cosh \xi) S e_{n}(k f, \cos \theta), & n \geq 0 \text { (for even mode) } \\ \operatorname{Ro}_{n}^{(3)}(k f, \cosh \xi) S o_{n}(k f, \cos \theta), & n \geq 1(\text { for odd mode) }\end{cases}
$$

where $k$ is a positive real number representing the wavenumber, $\mathrm{Se}_{n}\left(\mathrm{resp} . \mathrm{So}_{n}\right)$ are the even (resp. odd) periodic Mathieu functions and $\operatorname{Re}_{n}^{(3)}$ (resp. $\operatorname{Ro}_{n}^{(3)}$ ) are the even (resp. odd) radial Mathieu functions of the third kind (see p. 376 in [28]). In addition, the periodic functions $\mathrm{Se}_{n}$ and $\mathrm{So}_{n}$ satisfy the differential equation (see Eq. (15)-(16), p. 377 in [10]):

$$
\frac{\partial^{2} v_{n}}{\partial \theta^{2}}+\left(c_{n}-\frac{(k f)^{2}}{2} \cos ^{2} \theta\right) v_{n}=0
$$

where $c_{n}$ is called the characteristic value (see p. 376 in [10] or p. 721 in [1]). For even modes i.e. $v_{n}=\mathrm{Se}_{n}$, we have $c_{n}=a_{n}:=a_{n}(k f)$, and for odd modes i.e. $v_{n}=\mathrm{So}_{n}$, we have $c_{n}=b_{n}:=b_{n}(k f)$.

\subsection{The new two-dimensional approximate local DtN boundary conditions}

The two-dimensional first- and second-order approximate local DtN boundary conditions, defined on the elliptical-shaped surface $\xi=\xi_{0}$, are given by:

$$
\begin{gathered}
(\mathrm{DtN} 1) \quad \frac{\partial u}{\partial \xi}=\frac{\sqrt{1-e^{2}}}{e} r_{0} u \\
(\mathrm{DtN} 2) \frac{\partial u}{\partial \xi}=\frac{\sqrt{1-e^{2}}}{\left(a_{0}-a_{1}\right) e}\left[\left(a_{0} r_{1}-a_{1} r_{0}-\left(r_{0}-r_{1}\right) \frac{(e k a)^{2}}{2} \cos 2 \theta\right) u+\left(r_{1}-r_{0}\right) \frac{\partial^{2} u}{\partial \theta^{2}}\right]
\end{gathered}
$$

where the coefficient $r_{n}$ is given by:

$$
r_{n}=\frac{\frac{\partial \operatorname{Re}_{n}^{(3)}}{\partial \xi}\left(e k a, e^{-1}\right)}{\operatorname{Re}_{n}^{(3)}\left(e k a, e^{-1}\right)} ; n \geq 0
$$

It is worthwhile to note the following:

i. The Robin-type boundary conditions (DtN1) and (DtN2) given by Eqs. (4)(5) are, by construction, exact representations of the first elliptical mode $u_{0}$ given by Eq. (2). In addition, the boundary condition (DtN2) is an exact representation of the second even mode $u_{1}$ given by Eq. (2). The detailed derivation of these two absorbing boundary conditions can be found 
in $[4,26]$. Furthermore, Reference [26] contains the construction of a secondorder approximate DtN boundary condition that is exact for the first mode $u_{0}$ and the second odd mode $u_{1}$, as well as a local boundary condition that is exact, in the least-squares sense, for the first mode $u_{0}$, the second even mode $u_{1}$, and the second odd mode $u_{1}$. Since the performance of all these three approximate local DtN conditions are comparable if not identical, as demonstrated in [26], we report in this paper only on the performance of the absorbing boundary conditions DtN1 and DtN2 given by Eqs. (4)-(5).

ii. The local feature of the absorbing boundary conditions DtN1 and DtN2 given by Eqs. (4)-(5) is of a great interest from a numerical view point. Indeed, the incorporation of these conditions in any finite element code introduces only mass- and stiffness-type matrices defined on the exterior boundary. The computation of these matrices can achieved easily by evaluating the coefficients $a_{n}$ and $r_{n}$ once for all at a pre-processing level.

iii. The absorbing boundary conditions DtN1 and DtN2 given by Eqs. (4)(5) become identical, as $e \rightarrow 0$ (the ellipse becomes a circle), to the twodimensional approximate local DtN boundary conditions designed for circularshaped boundaries $[17,18]$. This property can be easily established using the asymptotic behavior of the even radial Mathieu functions of the third kind $\operatorname{Re}_{n}^{(3)}$ and the even characteristic values $a_{n}$.

\subsection{Performance analysis for two-dimensional radiating problems}

We analyze in this paragraph the effect of low wavenumber $k a$ and eccentricity $e$ on the performance of DtN1 and DtN2 given by Eqs. (4)-(5) in the case of radiating problems. Similarly to $[18,24,25]$, we assess the performance of these absorbing boundary conditions in the context of the OSRC formulation using the specific impedance introduced in $[11,12]$. This non-dimensional quantity measures the effect of the truncated medium in physical terms. It provides a practical tool for evaluating the performance of a given approximate representation. In the elliptical coordinates system, the specific impedance can be expressed as follows $[24,25]$ :

$$
Z=\left.\frac{i \sqrt{1-e^{2}} k a u}{\frac{\partial u}{\partial \xi}}\right|_{\xi=\xi_{0}}
$$

Moreover, we recall that the two-dimensional exact specific impedance $Z_{n}^{\text {ex2 }}$ for the $n^{\text {th }}$ even mode, on the boundary of an ellipse at $\xi=\xi_{0}$, is given by (see Eq. (13), p. 3626 in [24]):

$$
Z_{n}^{\mathrm{ex} 2}=\frac{\mathrm{i} \sqrt{1-e^{2}} k a \operatorname{Re}_{n}^{(3)}\left(e k a, e^{-1}\right)}{\frac{\partial \operatorname{Re}_{n}^{(3)}}{\partial \xi}\left(e k a, e^{-1}\right)}=\frac{\mathrm{i} e k a}{r_{n}}
$$


where the coefficient $r_{n}$ is given by Eq. (6). Furthermore, its asymptotic behavior, as $k a \rightarrow 0$, is given by (see Eq. (17), p. 3627 in [24]) :

$$
Z_{n}^{\mathrm{ex} 2} \sim \begin{cases}\pi \frac{k a}{2}+\mathrm{i} k a \ln (k a) & \text { if } n=0 \\ \frac{4 \pi}{(n !)^{2}}\left(\frac{k a}{2}\right)^{(2 n+1)}-\mathrm{i} \frac{k a}{n} & \text { if } n \geq 1\end{cases}
$$

The following Lemma states the expressions of the approximate specific impedances for the $n^{\text {th }}$ even elliptical mode, on the surface of an ellipse at $\xi=\xi_{0}$. This lemma results, as demonstrated in [26], from substituting $u=u_{n}$ in Eq. (7), and from using the absorbing boundary conditions given by Eqs. (4)(5) to evaluate $\frac{\partial u_{n}}{\partial \xi}$

Lemma 1 The two-dimensional approximate specific impedance $Z_{n}^{\mathrm{DtN1}, 2 \mathrm{~d}}$, for the $n^{\text {th }}$ even elliptical mode, corresponding to DtN1 absorbing boundary condition is given by:

$$
Z_{n}^{\mathrm{DtN} 1,2 \mathrm{~d}}=Z_{0}^{\mathrm{ex} 2} ; \quad \forall n \geq 0
$$

The two-dimensional approximate specific impedance $Z_{n}^{\mathrm{DtN2} 2 \mathrm{~d}}$, for the $n^{\text {th }}$ even elliptical mode, corresponding to DtN2 absorbing boundary condition is given by:

$$
Z_{n}^{\mathrm{DtN} 2,2 \mathrm{~d}}=\frac{1}{\frac{1}{Z_{0}^{\mathrm{ex} 2}}+\frac{a_{0}-a_{n}}{a_{0}-a_{1}}\left[\frac{1}{Z_{1}^{\mathrm{ex} 2}}-\frac{1}{Z_{0}^{\mathrm{ex} 2}}\right]} ; \quad \forall n \geq 0
$$

where $a_{n}:=a_{n}(k f)$ is the characteristic value corresponding to the $n^{\text {th }}$ even periodic Mathieu function $S_{n}$ (see p. 376 in [28] or p. 721 in [1]).

Remark 2 By construction, we have $Z_{0}^{\mathrm{DtN} 1,2 \mathrm{~d}}=Z_{0}^{\mathrm{DtN} 2,2 \mathrm{~d}}=Z_{0}^{\text {ex2 }}$, and $Z_{1}^{\mathrm{DtN} 2,2 \mathrm{~d}}=$ $Z_{1}^{\text {ex2 }}$. Furthermore, when $e \rightarrow 0$, i.e. the ellipse becomes a circle, the twodimensional approximate DtN specific impedances given by Eqs. (10)-(11) are identical to the ones obtained in the case of circular radiators [18].

The next proposition states the asymptotic behavior, as $k a \rightarrow 0$, of the twodimensional approximate specific impedance $Z_{n}^{\mathrm{DtN2,2 \textrm {d }}}$.

Proposition 2.1 The asymptotic behavior, as $k a \rightarrow 0$, of the two-dimensional approximate DtN2 specific impedance for the $n^{\text {th }}$ elliptical even mode is given by:

$$
Z_{n}^{\mathrm{DtN} 2,2 \mathrm{~d}} \sim \frac{\pi}{2 n^{2}}(k a)^{3}-\mathrm{i} \frac{k a}{n^{2}} ; \quad n \geq 2
$$

Proof of Proposition 2.1. We recall that, as $k a \rightarrow 0$, the asymptotic behavior of the characterisitic values $a_{n}$ (see Eq. (36) p. 120 in [23]) is given 
by:

$$
a_{n} \sim \begin{cases}-\frac{(e k a)^{4}}{32} & \text { if } n=0 \\ n^{2} & \text { if } n \geq 1\end{cases}
$$

Hence, as $k a \rightarrow 0$, we substitute Eq. (9) and Eq. (13) into Eq. (11) and deduce that $Z_{n}^{\mathrm{DtN} 2,2 \mathrm{~d}} \sim \phi_{n}(k a)(n \geq 2)$, where:

$$
\phi_{n}(k a)=\frac{1}{\frac{1}{\pi \frac{k a}{2}+\mathrm{i} k a \ln (k a)}+\frac{-\frac{(e k a)^{4}}{32}-n^{2}}{-\frac{(e k a)^{4}}{32}-1}\left[\frac{1}{\frac{\pi}{2}(k a)^{3}-\mathrm{i} k a}-\frac{1}{\pi \frac{k a}{2}+\mathrm{i} k a \ln (k a)}\right]}
$$

Therefore, Eq. (12) is an immediate consequence of the asymptotic behavior, as $k a \rightarrow 0$, of $\phi_{n}(k a)$.

Remark 3 First, observe that the asymptotic behavior, as $k a \rightarrow 0$, of $Z_{n}^{\mathrm{DtN1}, 2 \mathrm{~d}}$ is identical to the behavior of the exact specific impedance $Z_{0}^{\text {ex2 }}$ given by Eq. (9). In addition, for $\mathrm{n}=0,1$, the asymptotic behavior of $Z_{n}^{\mathrm{DtN} 2,2 \mathrm{~d}}$ is, by construction, identical to the behavior of the exact specific impedance $Z_{n}^{\text {ex2 }}$. Second, for $n \geq 2$, the asymptotic behavior of $Z_{n}^{\mathrm{DtN} 2,2 \mathrm{~d}}$ is identical to the case of a circle (see Eq.(62), p. 27 in [18]) (independent of the eccentricity e). Last, the asymptotic behavior given by Eq. (9) and Eq. (12) indicate that for higher modes $(n \geq 2)$, the real part of the exact impedance tends to zero, as $k a \rightarrow 0$, faster than the DtN2 specific impedance. This result suggests that, similarly to BGT2 boundary condition, the two-dimensional DtN2 boundary condition may not be appropriate for single higher elliptical modes.

Next, we investigate numerically the performance of the designed approximate local DtN boundary conditions when computing two-dimensional single higher order elliptical modes $(n \geq 2)$. Recall that by construction DtN2 is exact for the first two even modes $(n=0,1)$, and therefore outperforms BGT2 expressed in elliptical coordinates (see Eq. (11) p. 3626 in Reference [24]). We have performed several experiments to assess the effect of the wavenumber and the slenderness of the boundary on the performance of the second-order DtN boundary condition DtN2 given by Eq. (5). All the obtained results are reported in $[4,26]$. These results indicate, as demonstrated analytically, that overall both DtN2 and BGT2 absorbing boundary conditions perform poorly when computing higher elliptical modes $(n \geq 2)$. This conclusion is clearly illustrated by the numerical results depicted in Figs. (1)-(2). These results represent the relative errors when computing the second even elliptical mode $(n=2)$, for two wavenumber values: $k a=0.1$ (see Fig. (1)) and $k a=1$ (see Fig. (2)). These results have been obtained for six eccentricity values $e=0.1,0.2$ corresponding to an elliptical-shaped boundary "close" to a circle, 
$e=0.4,0.6$ corresponding to a "regular" elliptical boundary, and $e=0.8,0.9$ corresponding to a "very" elongated elliptical boundary. Note that we have reported the obtained results for the relative errors as a function of $\theta$ since the approximate BGT2 specific impedance depends on $\theta \in[0,2 \pi)$ (see Eq. (15) p. 3627 in [24]). Observe that for low frequency (see Fig. (1)) the relative error is about $20 \%$ for all eccentricity values and observation angle $\theta$. The situation is slightly different for $k a=1$. Indeed, one can observe (see Fig. (2)) that DtN2 boundary condition retains an acceptable level of accuracy (the relative error is less that $10 \%)$ for very elongated boundaries $(e=0.8,0.9)$ while BGT2 boundary condition exhibits similar behavior when the boundary is close to a circle $(e=0.1,0.2)$. Note that the level of accuracy deteriorates significantly and both conditions perform poorly when computing higher order elliptical modes as expected and observed in $[4,26]$. 

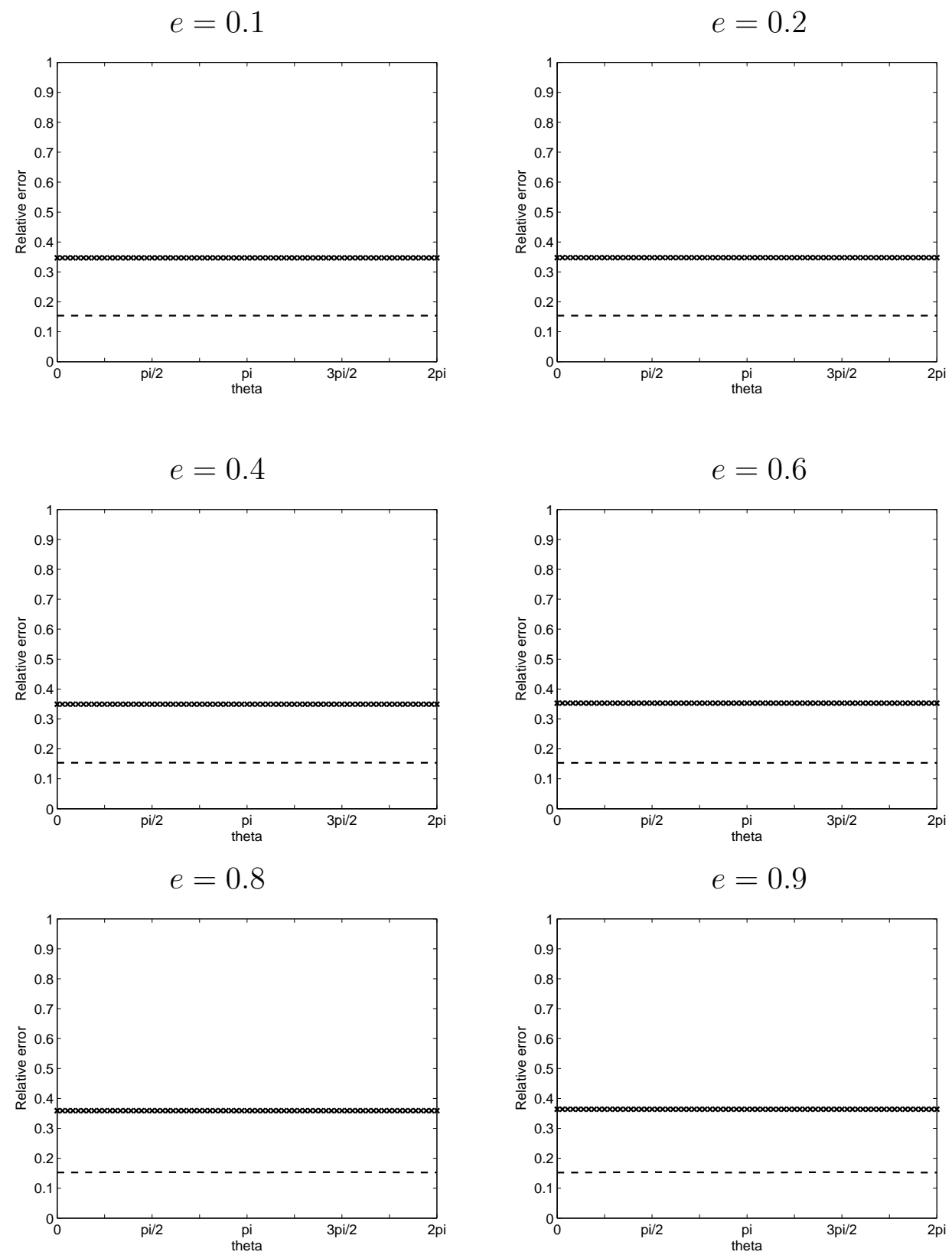

Fig. 1. Relative error of the specific impedance for DtN2 (crossed), BGT2 (dashed) when computing the second even elliptical mode $(\mathrm{n}=2)$ for $k a=0.1$. 

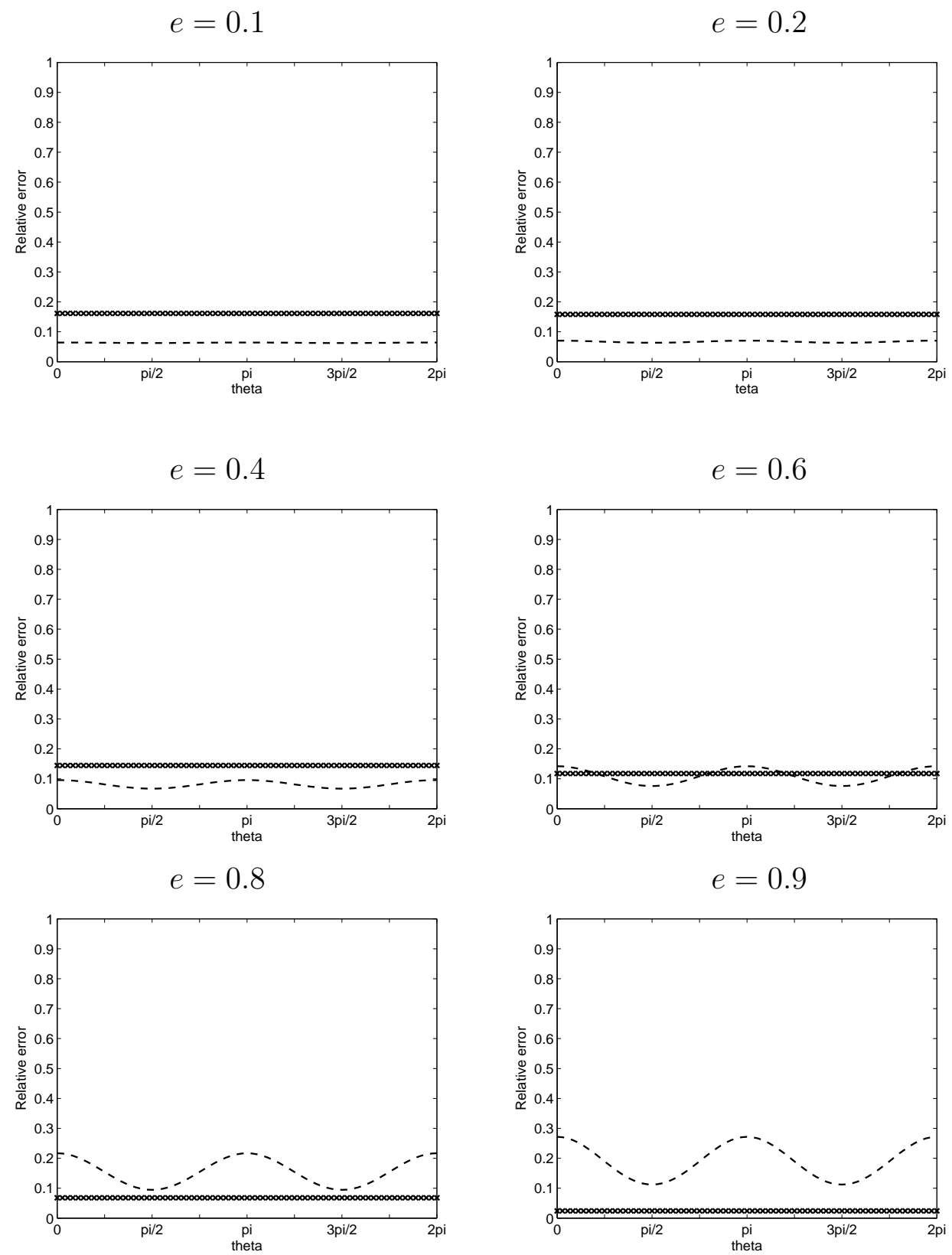

Fig. 2. Relative error of the specific impedance corresponding to DtN2 (crossed), BGT2 (dashed) when computing the second even elliptical mode $(\mathrm{n}=2)$ for $k a=1$. 
This paragraph is devoted to the analysis of the effect of the wavenumber and eccentricity values on the performance of the approximate local DtN absorbing boundary conditions given by Eqs. (4)-(5) when applied for solving two-dimensional acoustic scattering problems by sound-soft obstacles. We assume the scatterer to be a rigid ellipse, and the incident field $u^{\text {inc }}$ to be a plane wave with incident angle $\theta_{0}$ given, in elliptical coordinates, by:

$$
u^{\mathrm{inc}}=\mathrm{e}^{\mathrm{i} k f \cosh \xi\left(\cos \theta \cos \theta_{0}+\tanh \xi \sin \theta \sin \theta_{0}\right)}
$$

Therefore, the acoustic scattered field $u^{\text {scat }}$ can be represented by the following series [7]:

$$
\begin{aligned}
u^{\mathrm{scat}}=-\sqrt{8 \pi}( & \sum_{m=0}^{\infty} \mathrm{i}^{m} K e_{m} R e_{m}^{(3)}(k f, \cosh \xi) S e_{m}(k f, \cos \theta) \\
& \left.+\sum_{m=1}^{\infty} \mathrm{i}^{m} K o_{m} R o_{m}^{(3)}(k f, \cosh \xi) S o_{m}(k f, \cos \theta)\right)
\end{aligned}
$$

where

$$
\begin{aligned}
K e_{m} & =\frac{1}{N_{m}^{(e)}} \frac{\operatorname{Re}_{m}^{(1)}\left(e k a, e^{-1}\right) \operatorname{Se}_{m}\left(e k a, \cos \theta_{0}\right)}{\operatorname{Re}_{m}^{(3)}\left(e k a, e^{-1}\right)} \\
K o_{m} & =\frac{1}{N_{m}^{(o)}} \frac{\operatorname{Ro}_{m}^{(1)}\left(e k a, e^{-1}\right) \operatorname{Se}_{m}\left(e k a, \cos \theta_{0}\right)}{\operatorname{Ro}_{m}^{(3)}\left(e k a, e^{-1}\right)}
\end{aligned}
$$

and $N_{m}^{(e)}$ (resp. $N_{m}^{(o)}$ ) is the normalization factor of the even (resp. odd) angular Mathieu functions (see Eq. (18), p. 377 in [28]).

Consequently, the exact specific impedance $Z^{\text {ex2 }}$ on the surface of an ellipse at $\xi=\xi_{0}$ is given by (see Eq. (33), p. 3635 in [24]):

$$
Z^{\mathrm{ex} 2}=-\frac{\mathrm{i} \sqrt{1-e^{2}} k a u^{\mathrm{inc}}}{\left.\frac{\partial}{\partial \xi}\left(u^{\mathrm{scat}}\right)\right|_{\xi=\xi_{0}}}
$$

and its asymptotic behavior, as $k a \rightarrow 0$, is given by (see Eq.(38), p. 3637 in $[24])$ :

$$
Z^{\mathrm{ex} 2} \sim Z_{0}^{\mathrm{ex} 2} \sim \pi \frac{k a}{2}+\mathrm{i} k a \ln (k a) ; \quad k a \rightarrow 0
$$

where $Z_{0}^{\text {ex2 }}$ is the exact impedance corresponding to the two-dimensional first mode (see Eq. (9)). The next lemma states the expressions of the approximate specific impedances on the boundary $\xi=\xi_{0}$ of an elliptical-shaped sound-soft scatterer. This lemma results, as demonstrated in [26], from a straightforward substitution into Eq. (7) of $u=-u^{\text {inc }}$ (sound-soft scatterer) and from using the 
approximate local DtN boundary conditions given by Eqs. (4)-(5) to evaluate $\frac{\partial u}{\partial \xi}=-\frac{\partial u^{\text {inc }}}{\partial \xi}$ at $\xi=\xi_{0}$.

Lemma 4 The two-dimensional approximate specific impedance $Z^{\mathrm{DtN1}, 2 \mathrm{~d}}$, for a sound-soft scattering problem, corresponding to DtN1 absorbing boundary condition is given by:

$$
Z^{\mathrm{DtN} 1,2 \mathrm{~d}}=Z_{0}^{\mathrm{ex} 2}
$$

The two-dimensional approximate specific impedance $Z^{\mathrm{DtN2} 2 \mathrm{~d}}$, for a soundsoft scattering problem, corresponding to DtN2 absorbing boundary condition is given by:

$$
Z^{\mathrm{DtN} 2,2 \mathrm{~d}}=\frac{1}{\frac{1}{Z_{0}^{\mathrm{ex} 2}}+\frac{1}{a_{0}-a_{1}}\left(\delta+a_{0}\right)\left(\frac{1}{Z_{1}^{\mathrm{ex} 2}}-\frac{1}{Z_{0}^{\mathrm{ex} 2}}\right)}
$$

where

$$
\begin{aligned}
& \delta=-i k a \lambda-(k a)^{2}\left(\frac{\partial \lambda}{\partial \theta^{2}}\right)-\frac{(e k a)^{2}}{2} \cos 2 \theta \\
& \lambda=\cos \theta \cos \theta_{0}+\sqrt{1-e^{2}} \sin \theta \sin \theta_{0}
\end{aligned}
$$

Remark 5 Note that when $e \rightarrow 0$, i.e. the ellipse becomes a circle, the approximate DtN specific impedances given by Eqs. (19) and (20) are identical to the ones obtained in the case of a circular-shaped scatterer [18].

The next proposition states the asymptotic behavior, as $k a \rightarrow 0$, of the twodimensional approximate specific impedance $Z^{\mathrm{DtN2,2d}}$.

Proposition 2.2 The asymptotic behavior, as $k a \rightarrow 0$, of the two-dimensional approximate DtN2 specific impedance for the sound-soft scattering problem is given by:

$$
Z^{\mathrm{DtN} 2,2 \mathrm{~d}} \sim Z_{0}^{\mathrm{ex} 2}
$$

Proof of Proposition 2.2. First, we rewrite the expression of $Z^{\mathrm{DtN2} 2 \mathrm{~d}}$ given by Eq. (20) as follows:

$$
Z^{\mathrm{DtN} 2,2 \mathrm{~d}}=Z_{0}^{\mathrm{ex} 2} \frac{1}{1+\frac{a_{0}+\delta}{a_{0}-a_{1}}\left[\frac{Z_{0}^{\mathrm{ex} 2}}{Z_{1}^{\mathrm{ex} 2}}-1\right]}
$$

Next, observe that $\delta$, given by Eq. (21), can be also expressed as follows

$$
\delta \sim-\mathrm{i} k a \lambda \quad \text { as } \quad k a \rightarrow 0
$$


Consequently, it follows from using the asymptotic behavior of the characteristic values, given by Eq.(13), that:

$$
a_{0}+\delta \sim-\mathrm{i} k a \lambda \quad \text { as } \quad k a \rightarrow 0
$$

In addition, it follows from using the asymptotic behavior of the characteristic values, given by Eq.(13), that:

$$
a_{1}-a_{0} \sim 1 \quad \text { as } \quad k a \rightarrow 0
$$

and from the asymptotic behavior of the two-dimensional exact specific impedance $Z_{n}^{\text {ex2}}$, given by Eq.(9), that:

$$
\frac{Z_{0}^{\text {ex2 }}}{Z_{1}^{\text {ex2 }}}-1 \sim \ln (k a)+\mathrm{i} \frac{\pi}{2} \quad \text { as } \quad k a \rightarrow 0
$$

Therefore, it follows from Eqs. (25)-(27) that:

$$
\frac{a_{0}+\delta}{a_{0}-a_{1}}\left[\frac{Z_{0}^{\mathrm{ex} 2}}{Z_{1}^{\mathrm{ex} 2}}-1\right] \rightarrow 0 \quad \text { as } \quad k a \rightarrow 0
$$

which concludes the proof of Proposition 2.2.

Remark 6 First, we note that, unlike the approximate specific impedance coresponding to the second-order BGT2 condition (see Eq.(40), p. 3637 in [24]), the asymptotic behavior, as $k a \rightarrow 0$, of $Z^{\mathrm{DtN2}, 2 \mathrm{~d}}$ is identical to the behavior of the exact specific impedance $Z^{\mathrm{ex} 2}$ (see Eq. (18)). In addition, when $e \rightarrow 0$, the asymptotic behaviors of both $Z^{\mathrm{DtN1,2d}}$ and $Z^{\mathrm{DtN2}, 2 \mathrm{~d}}$ are identical to the case of circular-shaped sound-soft scatterers.

Next we investigate numerically the effect of the wavenumber and the slenderness of the boundary on the performance of the approximate local DtN boundary conditions given by Eqs. (4)-(5) when applied for solving sound-soft scattering problems by elliptical-shaped obstacles. We have performed such investigation in the OSRC context [21] and have compared the results to the ones obtained with BGT2 condition when expressed in elliptical coordinates (see Figs. (9) to (13) in [24]). All obtained results are reported in [4,26]. For illustration purpose, we present the results for only two values of the wavenumber, $k a=0.1$ and 1 , corresponding to three different values of the incidence angle $\theta_{0}=0, \frac{\pi}{4}$, and $\frac{\pi}{2}$. These results have been obtained for six eccentricity values $e=0.1,0.2$ corresponding to an elliptical-shaped boundary "close" to a circle, $e=0.4,0.6$ corresponding to a "regular" elliptical boundary, and $e=0.8,0.9$ corresponding to a "very" elongated elliptical boundary. Note that since all the approximate specific impedances depend on the observation angle $\theta \in[0,2 \pi)$, we have reported in Figs. (3)-(8) the relative errors as a function of $\theta$. The results reported in Figs. (3)-(8) suggest the following four remarks: 
i. Unlike the radiating problem, DtN2 absorbing boundary condition retains an excellent level of accuracy when solving acoustic problems for low wavenumbers, as expected (see Proposition 2.2). In addition, the results reported in Figs. (3)-(8), clearly demonstrate that such good performance is not sensitive to the values of eccentricity $e$. These results suggest in particular that DtN2 absorbing boundary condition given by Eq. (5) is appropriate for elongated boundaries.

ii. DtN2 absorbing boundary condition clearly outperforms the second-order BGT2 absorbing boundary condition especially for high eccentricity values. Indeed, there is a significant loss of accuracy for the BGT2 boundary condition when $e \geq 0.6$ (BGT2 delivers relative errors larger than $40 \%$ while the errors with DtN2 remain below 2\%). This demonstrates that DtN2 absorbing boundary condition extends the range of satisfactory performance to all eccentricity values in the low frequency regime.

iii. For some particular observation angles, DtN1 boundary condition surprisingly performs better than DtN2 boundary condition (see Figs. (6)-(8)). Nevertheless, the total relative error (the average of the relative error over all observation angles) delivered by DtN1 absorbing boundary condition is significantly higher than the total relative error corresponding to DtN2 absorbing boundary condition.

iv. For some particular observation angles, DtN1 boundary condition also outperforms the BGT2 boundary condition (see Figs. (6)-(8)). This better performance is particularly visible for $e=0.9$ when $k a=1$. 
$e=0.1$

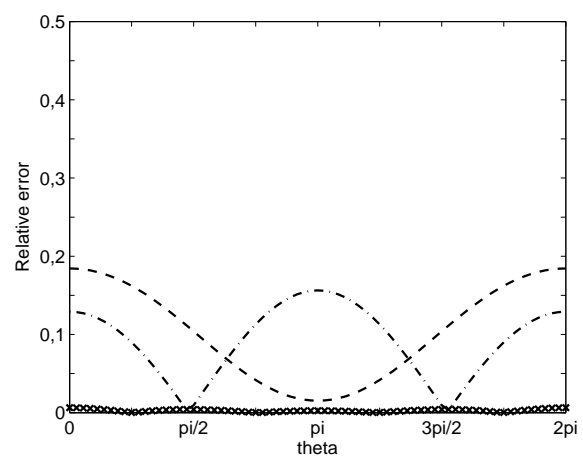

$e=0.4$

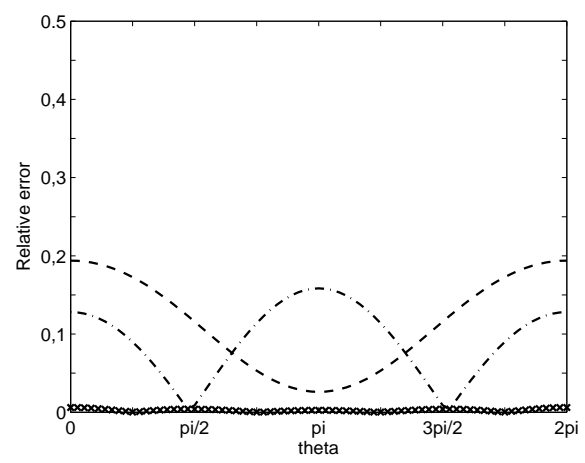

$e=0.8$

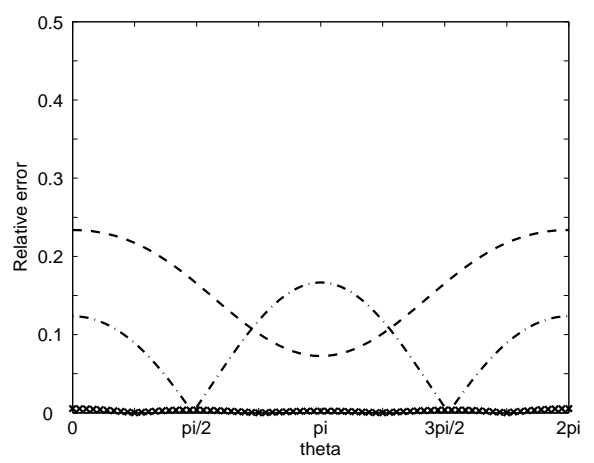

$e=0.2$

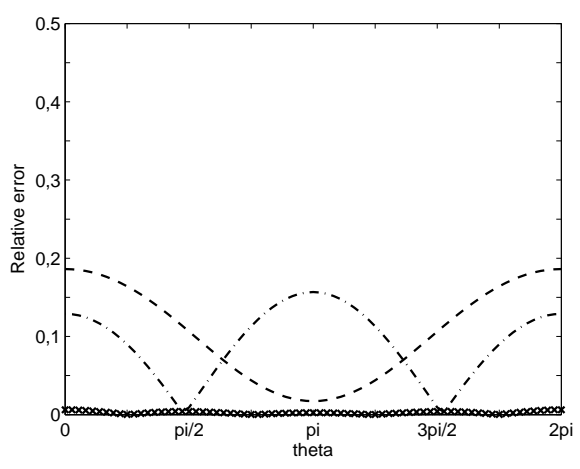

$e=0.6$

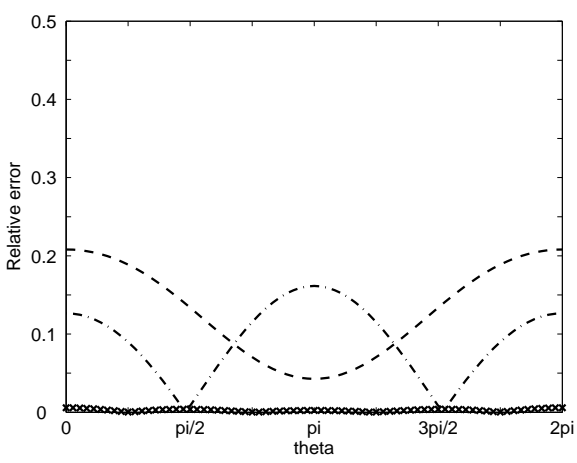

$e=0.9$

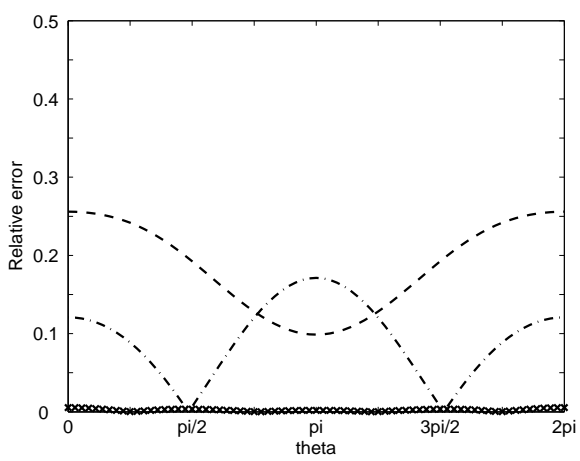

Fig. 3. Relative error of the specific impedance corresponding to DtN1 (dashed/dotted), DtN2 (crossed), BGT2 (dashed) when solving two-dimensional sound-soft scattering problem with $k a=0.1$ and incident angle $\theta_{0}=0$ (The relative error for DtN2 is below $2 \%$ ). 
$e=0.1$

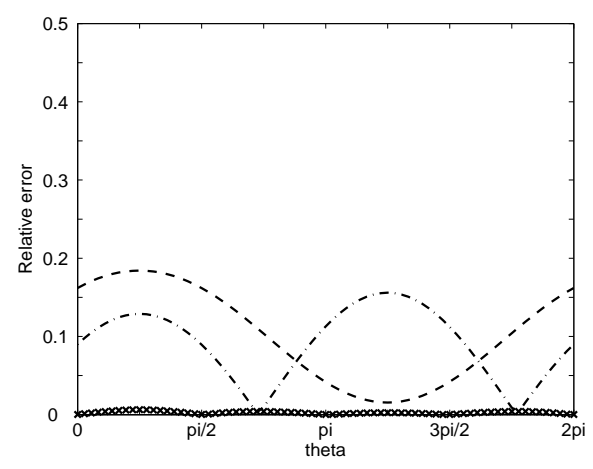

$e=0.4$

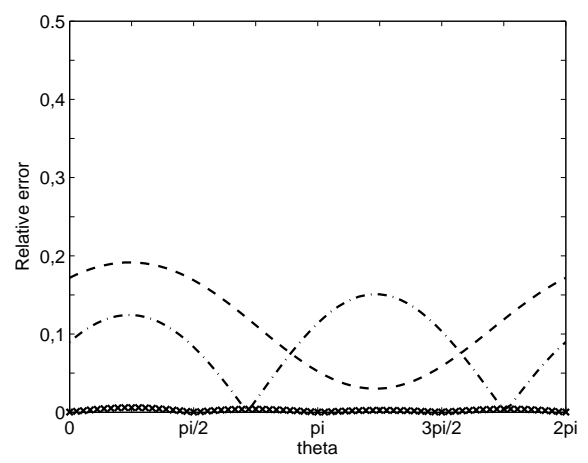

$e=0.8$

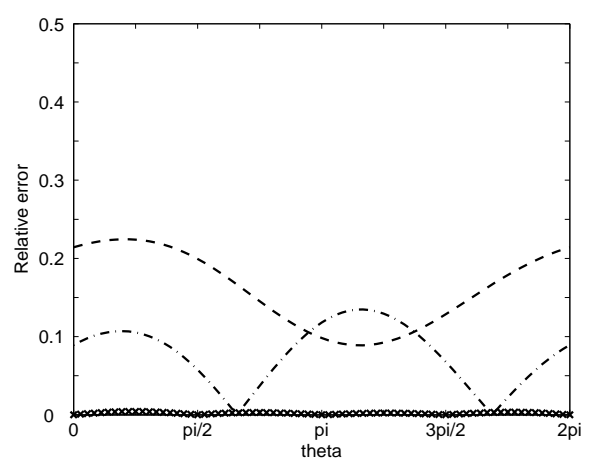

$e=0.2$

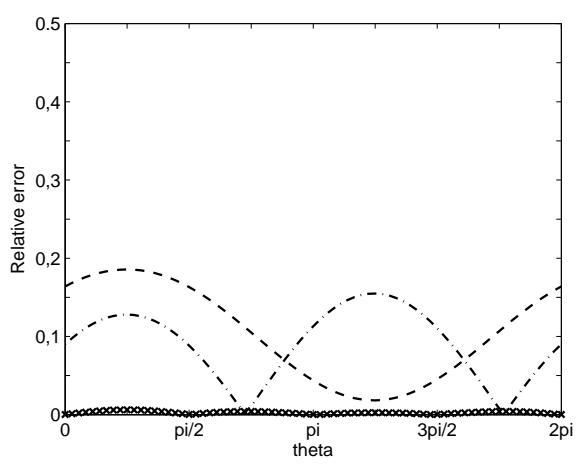

$e=0.6$

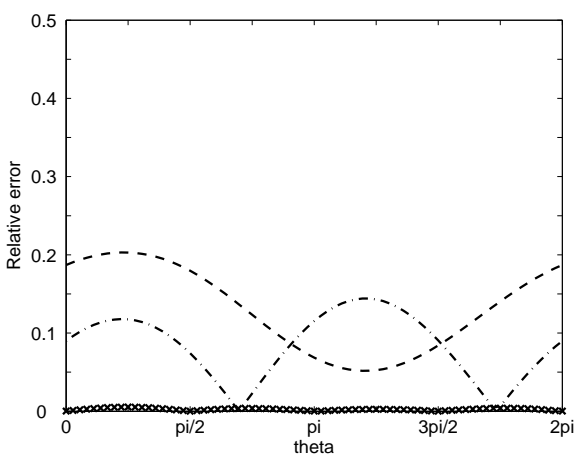

$e=0.9$

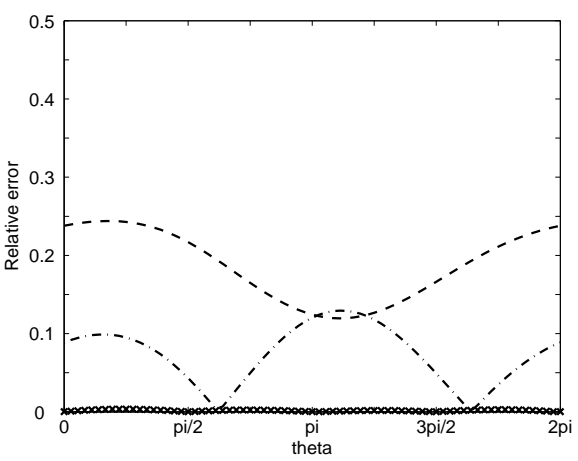

Fig. 4. Relative error of the specific impedance corresponding to DtN1 (dashed/dotted), DtN2 (crossed), BGT2 (dashed) when solving two-dimensional sound-soft scattering problem with $k a=0.1$ and incident angle $\theta_{0}=\frac{\pi}{4}$ (The relative error for DtN2 is below $2 \%$ ). 
$e=0.1$

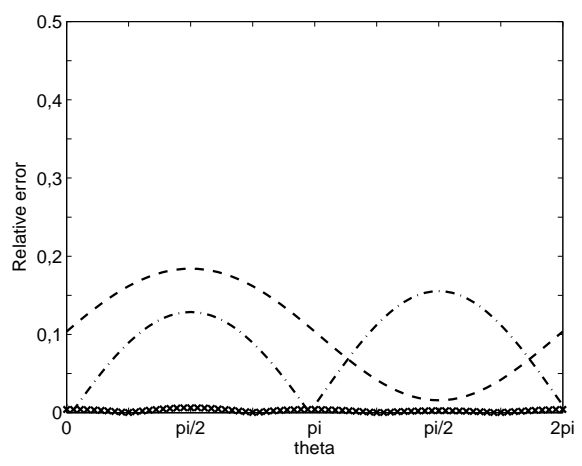

$e=0.4$

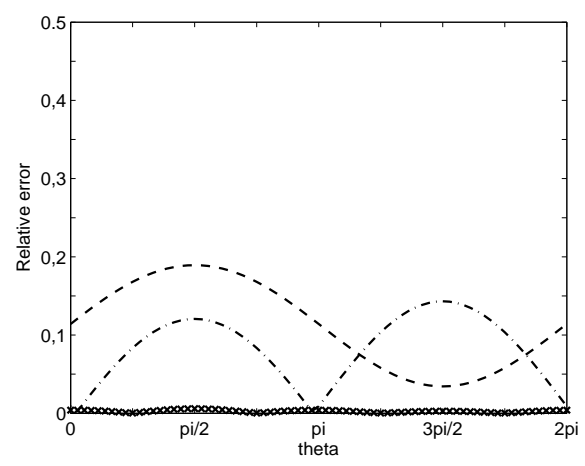

$e=0.8$

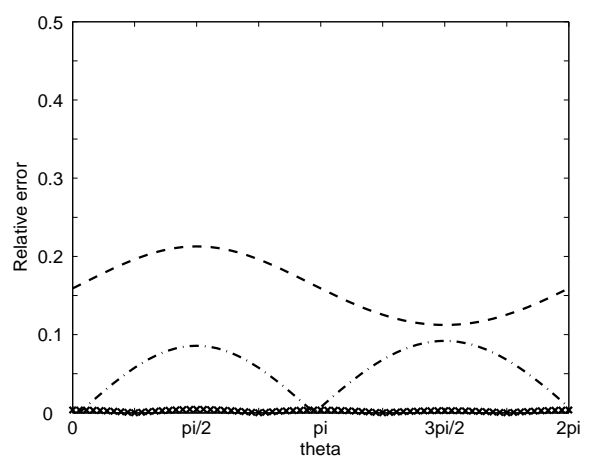

$e=0.2$

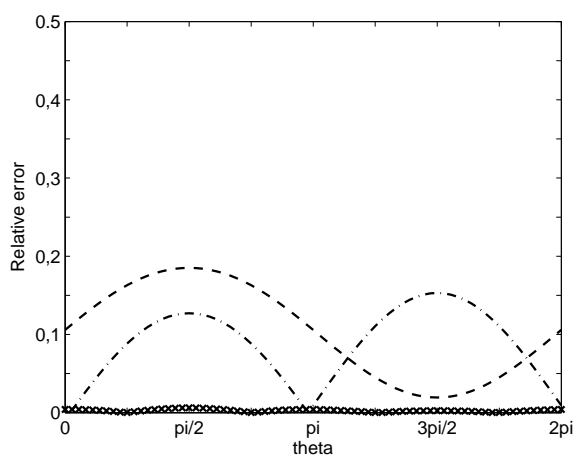

$e=0.6$

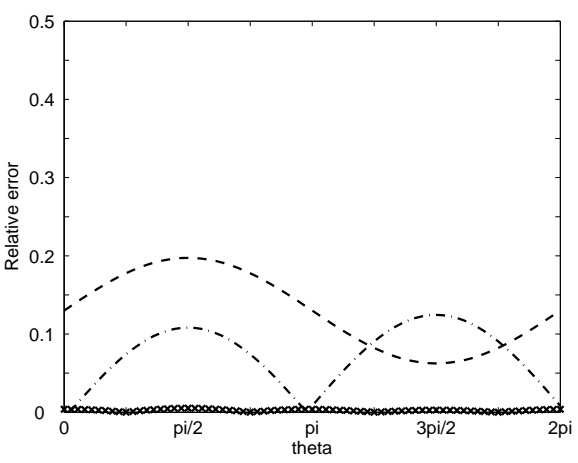

$e=0.9$

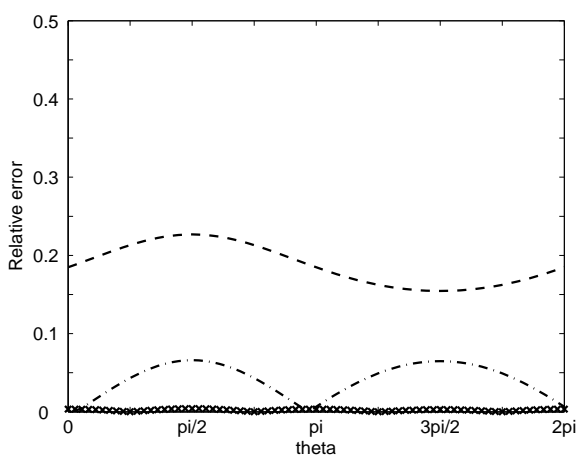

Fig. 5. Relative error of the specific impedance corresponding to DtN1 (dashed/dotted), DtN2 (crossed), BGT2 (dashed) when solving two-dimensional sound-soft scattering problem with $k a=0.1$ and incident angle $\theta_{0}=\frac{\pi}{2}$ (The relative error for DtN2 is below $2 \%$ ). 
$e=0.1$

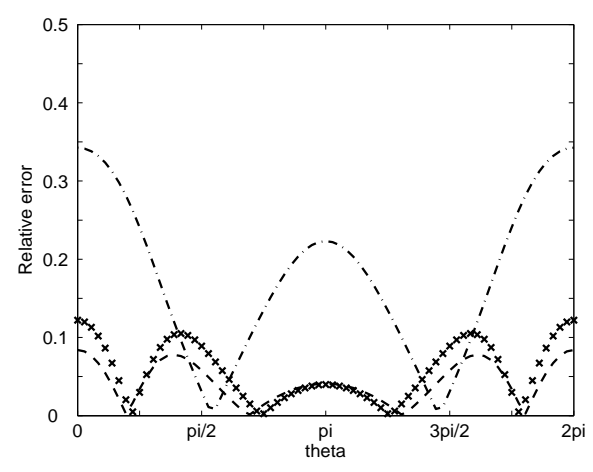

$e=0.4$

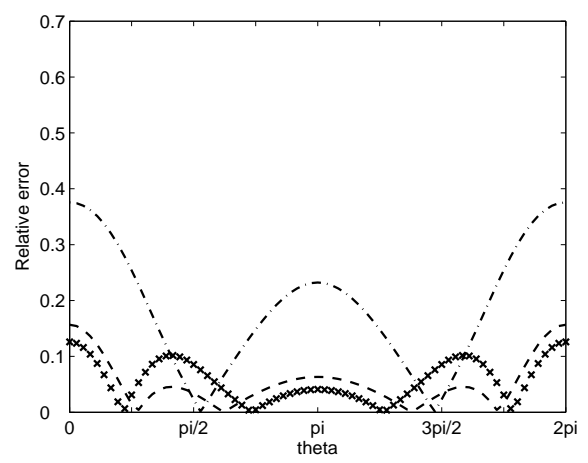

$e=0.8$

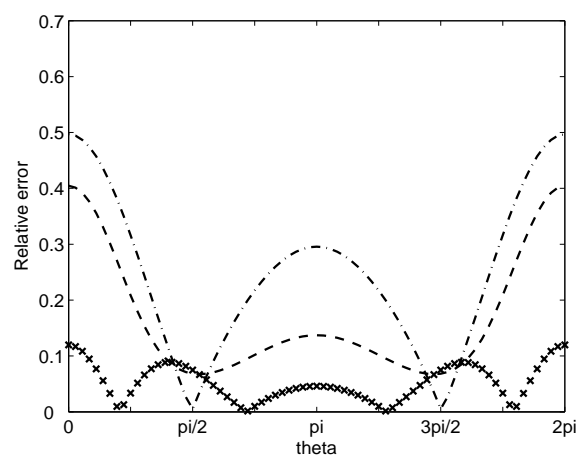

$e=0.2$

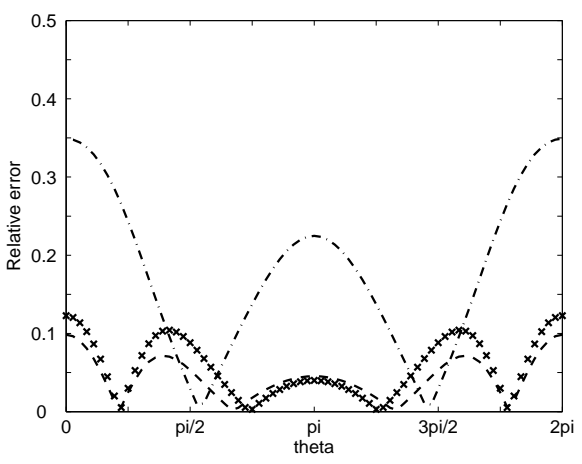

$e=0.6$

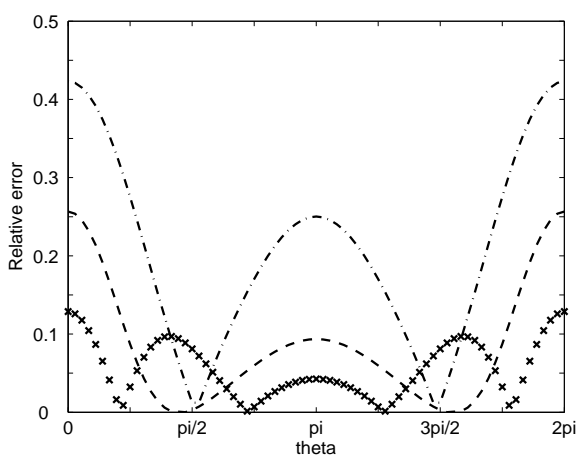

$e=0.9$

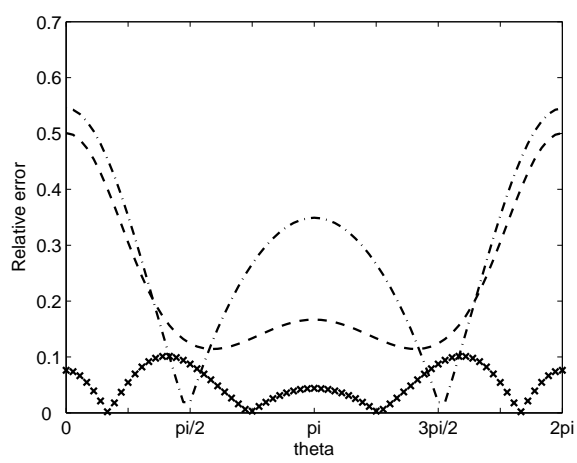

Fig. 6. Relative error of the specific impedance corresponding to DtN1 (dashed/dotted), DtN2 (crossed), BGT2 (dashed) when solving two-dimensional sound-soft scattering problem with $k a=1$ and incident angle $\theta_{0}=0$. 
$e=0.1$

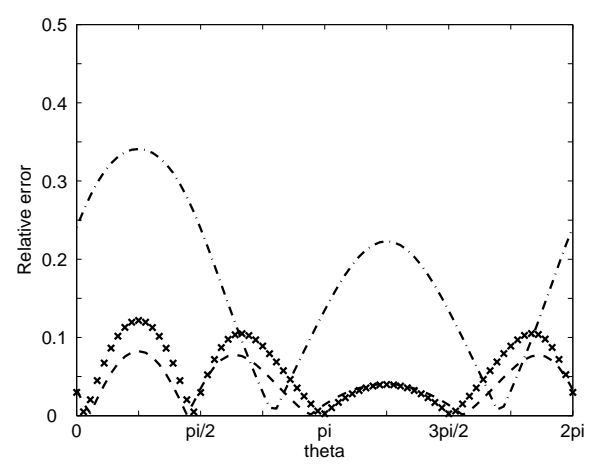

$e=0.4$

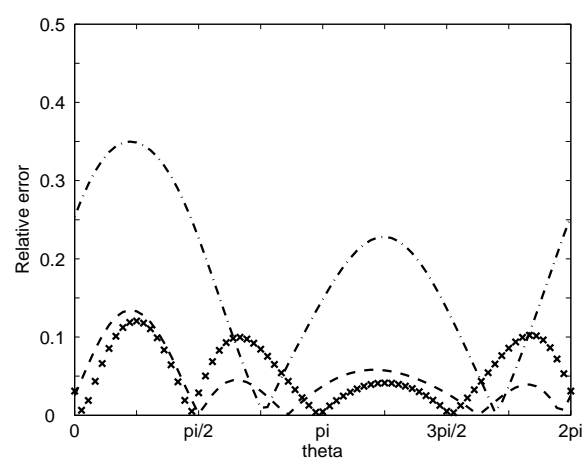

$e=0.8$

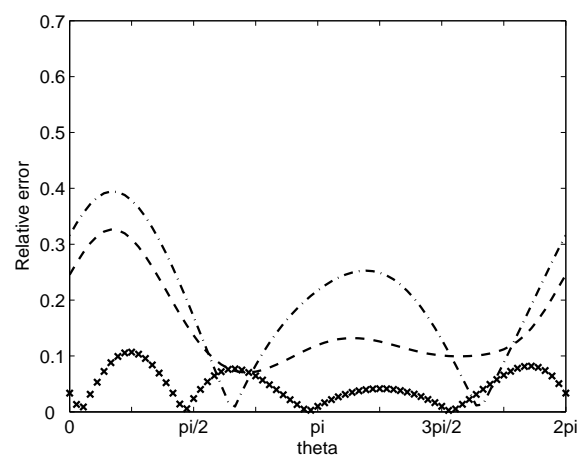

$e=0.2$

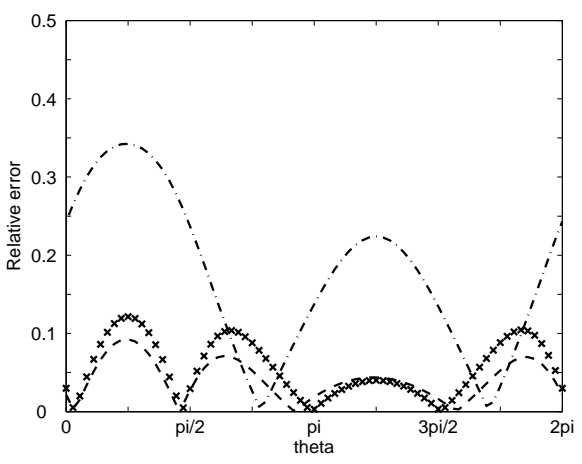

$e=0.6$

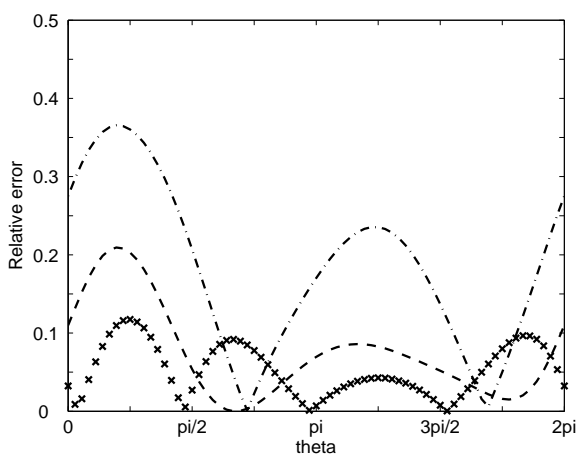

$e=0.9$

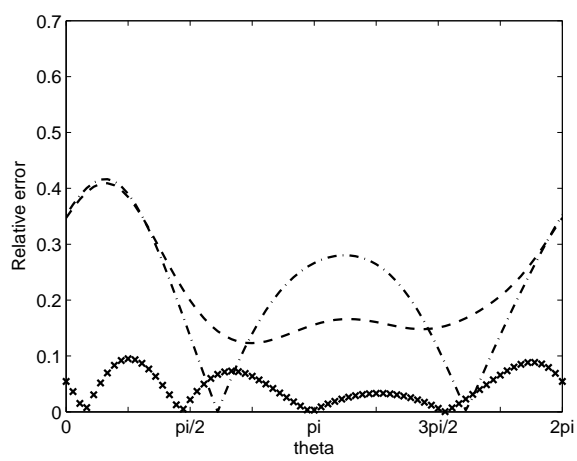

Fig. 7. Relative error of the specific impedance corresponding to DtN1 (dashed/dotted), DtN2 (crossed), BGT2 (dashed) when two-dimensional solving sound-soft scattering problem with $k a=1$ and incident angle $\theta_{0}=\frac{\pi}{4}$. 
$e=0.1$

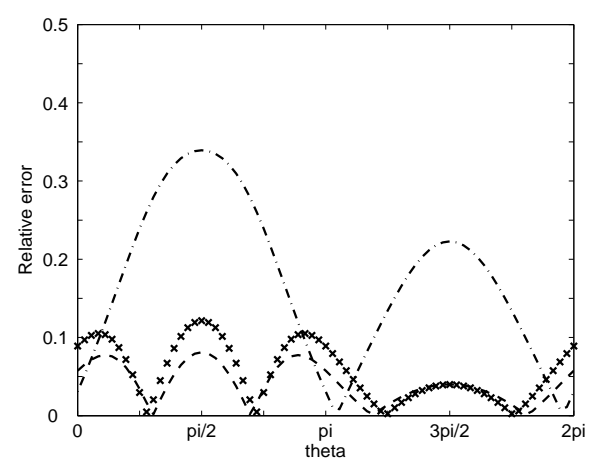

$e=0.4$

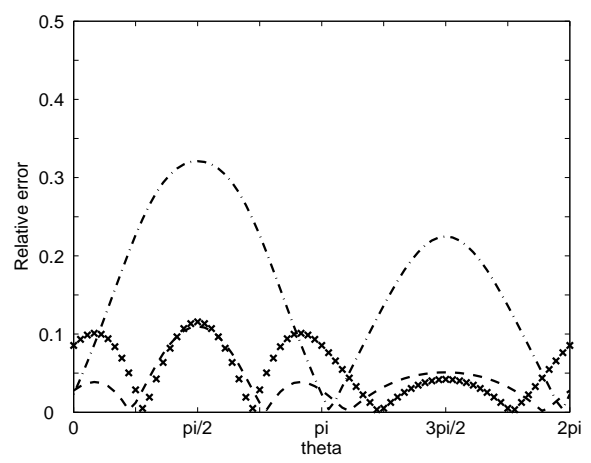

$e=0.8$

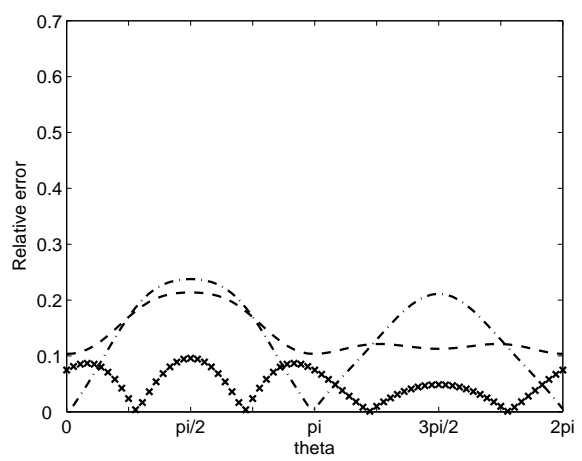

$e=0.2$

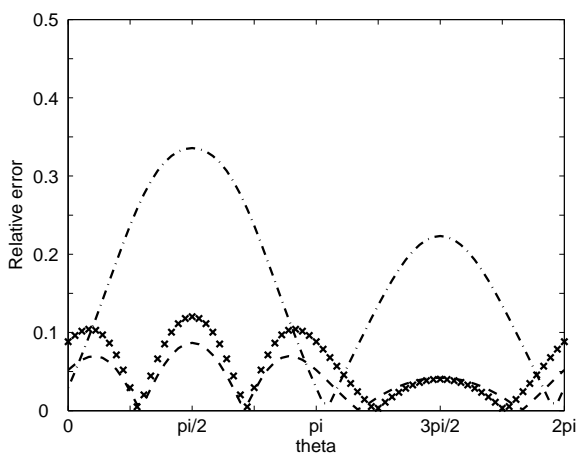

$e=0.6$

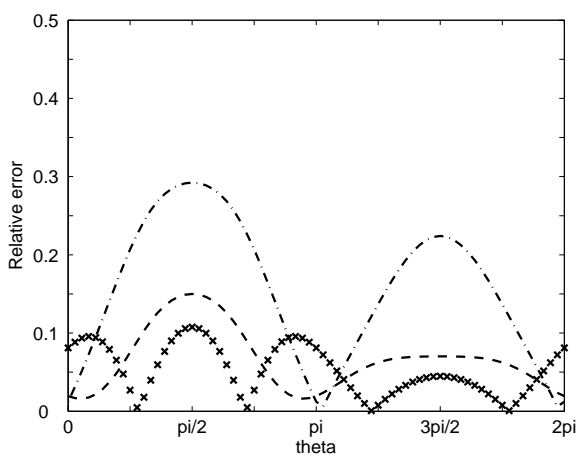

$e=0.9$

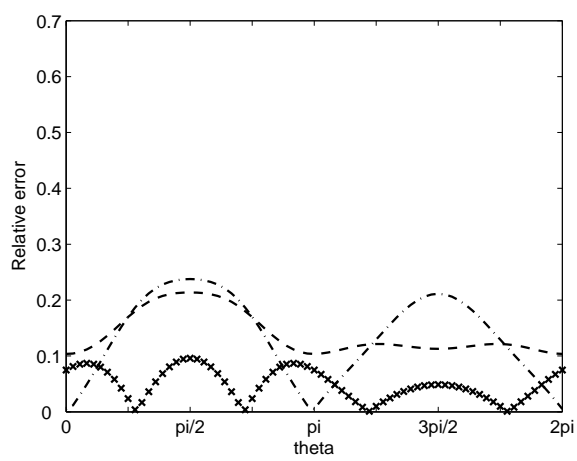

Fig. 8. Relative error of the specific impedance corresponding to DtN1 (dashed/dotted), DtN2 (crossed), BGT2 (dashed) when solving two-dimensional sound-soft scattering problem with $k a=1$ and incident angle $\theta_{0}=\frac{\pi}{2}$. 


\section{Three-dimensional Helmholtz problems}

We introduce in this section the three-dimensional approximate local DtN boundary conditions, and assess their performance when applied for solving low frequency radiating and scattering problems. More specifically, we investigate analytically and numerically the effect of low wavenumber and eccentricity on the performance of the constructed approximate local DtN boundary conditions in the context of the OSRC formulation.

\subsection{Preliminaries}

Throughout this section, we use the prolate spheroidal coordinates $(\xi, \varphi, \theta)$ which are related to the cartesian coordinates $(x, y, z)$ by $x=b \sin \varphi \cos \theta$, $y=b \sin \varphi \sin \theta$, and $z=a \cos \varphi$, where $\varphi \in[0, \pi)$ and $\theta \in[0,2 \pi)$. Similarly to the two-dimensional case, the parameters $a$ and $b$ respectively represent the major and the minor axes and are given by $a=f \cosh \xi$ and $b=f \sinh \xi ; \xi$ is a strictly positive real number and $f=\sqrt{a^{2}-b^{2}}$ is the interfocal distance. Finally, the eccentricity $e$ on the ellipse $\xi=\xi_{0}$ is given by Eq. (1). Note that when $e \rightarrow 0$ the prolate spheroid becomes a sphere, and the prolate spheroid degenerates into a line segment with length $2 f$ on the $z$-axis as $e \rightarrow 1$.

We recall that the $m n^{\text {th }}$ prolate spheroidal mode $u_{m n}(m \leq n)$ is given by [27]:

$$
u_{m n}=\mathrm{R}_{m n}^{(3)}(k f, \cosh \xi) \mathrm{S}_{m n}(k f, \cos \varphi) \cos m \theta
$$

where the functions $\mathrm{R}_{m n}^{(3)}(k f, \cosh \xi)$ are the radial spheroidal wave functions of the third kind and $\mathrm{S}_{m n}(k f, \cos \varphi)$ are the angular spheroidal wave functions. In addition, we have [10]:

$$
\frac{\partial}{\partial \varphi}\left(\sin \varphi \frac{\partial \mathrm{S}_{m n}}{\partial \varphi}\right)+\sin \varphi\left(\lambda_{m n}-(k f)^{2} \cos ^{2} \varphi-\frac{m^{2}}{\sin ^{2} \varphi}\right) \mathrm{S}_{m n}(k f, \cos \varphi)=0
$$

where $\lambda_{m n}:=\lambda_{m n}(k f)$ are the prolate spheroidal eigenvalues (see p.11 in [10]).

\subsection{The new three-dimensional approximate local boundary conditions}

The three-dimensional first- and second-order approximate local DtN boundary conditions, defined on the elliptical-shaped surface $\xi=\xi_{0}$, are given by: 


$$
\begin{gathered}
(\mathrm{DtN} 1) \quad \frac{\partial u}{\partial \xi}=\frac{\sqrt{1-e^{2}}}{e} R_{00} u \\
\text { (DtN2) } \frac{\partial u}{\partial \xi}=\frac{\sqrt{1-e^{2}}}{\left(\lambda_{01}-\lambda_{00}\right) e}[ \\
\left(\lambda_{01} R_{01}-\lambda_{00} R_{00}-\left(R_{00}-R_{01}\right)(e k a)^{2} \cos ^{2} \varphi\right) u \\
\left.+\left(R_{00}-R_{01}\right) \Delta_{\Gamma} u\right]
\end{gathered}
$$

where the coefficient $R_{m n}$ is given by:

$$
R_{m n}=\frac{\frac{\partial \mathrm{R}_{m n}^{(3)}}{\partial \xi}\left(e k a, e^{-1}\right)}{\mathrm{R}_{m n}^{(3)}\left(e k a, e^{-1}\right)} ; n \geq 0 \text { and } m \leq n
$$

and $\Delta_{\Gamma}$ denotes the Laplace Beltrami operator which reads in prolate spheroidal coordinates $(\xi, \varphi, \theta)$ as:

$$
\Delta_{\Gamma}=\frac{1}{\sin \varphi} \frac{\partial}{\partial \varphi}\left(\sin \varphi \frac{\partial}{\partial \varphi}\right)+\frac{1}{\sin ^{2} \varphi} \frac{\partial^{2}}{\partial \theta^{2}}
$$

It is worthwhile to note the following:

i. First, as stated earlier in the introduction, the construction methodology we propose for deriving the class of approximate local DtN boundary conditions in prolate spheroidal coordinates can be viewed as an inverse-type approach. More specifically, we start from a Robin-type boundary condition with unknown coefficients. Hence, in the case of DtN2 condition, we set:

$$
\frac{\partial u}{\partial \xi}=A u+B\left(\Delta_{\Gamma}-(e k a)^{2} \cos ^{2} \varphi\right) u
$$

where $A$ and $B$ are constant (independent of $\varphi$ ) to be determined. Note that, unlike DtN2 boundary condition for the spherical-shaped boundaries, the coefficients of this condition depend on the angular variable $\varphi$. Such dependence is necessary for constructing a symmetric boundary condition since the angular spheroidal wave functions satisfied differential given by Eq. (30). Then, we observe that all radiating modes $u_{m n}$ given by Eq. (29) satisfy

$$
\Delta_{\Gamma} u_{m n}=\left(-\lambda_{m n}+(e k a)^{2} \cos ^{2} \varphi\right) u_{m n}
$$

Hence, in order to determine the constants $A$ and $B$, we assume that, at $\xi=\xi_{0}$, we have:

$$
\frac{\partial u_{m n}}{\partial \xi}=A u_{m n}+B\left(\Delta_{\Gamma}-(e k a)^{2} \cos ^{2} \varphi\right) u_{m n} ; \quad m=0 \quad \text { and } \quad n=0,1
$$


Then, using Eq. (36), it follows that $(A, B)$ is the unique solution of the folllowing $2 \times 2$ linear system:

$$
\left\{\begin{array}{l}
A-B \lambda_{00}=\frac{\sqrt{1-e^{2}}}{e} R_{00} \\
A-B \lambda_{01}=\frac{\sqrt{1-e^{2}}}{e} R_{01}
\end{array}\right.
$$

where the coefficients $R_{m n}$ are given by Eq. (33).

The DtN2 boundary condition given by Eq. (32) is a direct consequence of solving the system (38) and substituting the expressions of $(A, B)$ into Eq. (35).

ii. The incorporation of the absorbing boundary conditions DtN1 and DtN2 given by Eqs. (31)-(32) in any finite element code introduces only mass- and stiffness-type matrices defined on the exterior boundary. The coefficients $\lambda_{m n}$ and $R_{m n}$ can be computed once for all at the pre-processing level.

iii. Similarly to the two-dimensional case, the absorbing boundary conditions DtN1 and DtN2 given by Eqs. (31)-(32) become identical, as $e \rightarrow 0$ (the prolate spheroid becomes a sphere), to the three-dimensional approximate local DtN boundary conditions designed for spherical-shaped boundaries $[17,18]$. This property can be easily established using the asymptotic behavior of the radial spheroidal wave functions of the third kind $\mathrm{R}_{m n}^{(3)}$ and the prolate spheroidal eigenvalues $\lambda_{m n}$.

\subsection{Performance analysis for three-dimensional radiating problems}

We analyze in this paragraph the effect of low wavenumber $k a$ and eccentricity $e$ on the performance of DtN1 and DtN2 given by Eqs. (31)-(32) in the case of radiating problems. Similarly to $[18,24,25]$, we assess the performance of these absorbing boundary conditions in the context of the OSRC formulation using the specific impedance introduced first in $[11,12]$ and given by Eq. (7).

First, we recall that the three-dimensional exact specific impedance $Z_{m n}^{\mathrm{ex} 3}$ for the $m n^{\text {th }}$ prolate spheroidal mode, on the surface of a prolate spheroid at $\xi=\xi_{0}$ is given by (see Eq. (64), p. 3645 in [24]):

$$
Z_{m n}^{\mathrm{ex} 3}=\frac{\mathrm{i} \sqrt{1-e^{2}} k a \mathrm{R}_{m n}^{(3)}\left(e k a, e^{-1}\right)}{\frac{\partial \mathrm{R}_{m n}^{(3)}}{\partial \xi}\left(e k a, e^{-1}\right)}=\frac{\mathrm{i} e k a}{R_{m n}}
$$

where the coefficient $R_{m n}$ is given by Eq. (33). Moreover, its asymptotic be- 
havior, as $k a \rightarrow 0$, is given by (see Eq. (67), p. 3646 in [24]):

$$
Z_{m n}^{\operatorname{ex} 3} \sim\left(\frac{(k a)^{n+1}}{(n+1)(2 n-1) ! !}\right)^{2}-\mathrm{i} \frac{k a}{n+1} ; \quad \text { as } \quad k a \rightarrow 0
$$

where the double factorial is defined in [1] (see also p. 30, Eq. (74) in Remark 4.2 .2 in [18]).

The following lemma states the expressions of three-dimensional approximate specific impedances for the $m n$-th prolate spheroidal mode, on the surface of a prolate spheroid at $\xi=\xi_{0}$. This lemma results, as demonstrated in [26], from substituting $u=u_{m n}$ in Eq. (7) and using the absorbing boundary conditions given by Eqs. (31)-(32) to evaluate $\frac{\partial u_{m n}}{\partial \xi}$.

Lemma 7 The three-dimensional approximate specific impedance $Z_{m n}^{\mathrm{DtN1}, 3 \mathrm{~d}}$, for the $m n^{\text {th }}$ prolate spheroidal mode, corresponding to DtN1 boundary condition is given by:

$$
Z_{m n}^{\mathrm{DtN} 1,3 \mathrm{~d}}=Z_{00}^{\mathrm{ex} 3}
$$

The three-dimensional approximate specific impedance $Z_{m n}^{\mathrm{DtN} 2,3 \mathrm{~d}}$, for the $m n^{\text {th }}$ prolate spheroidal mode, corresponding to DtN2 boundary condition is given by:

$$
Z_{m n}^{\mathrm{DtN2} 23 \mathrm{~d}}=\frac{\lambda_{01}-\lambda_{00}}{\left(\lambda_{01}-\lambda_{m n}\right) \frac{1}{Z_{00}^{\text {ex3 }}}+\left(\lambda_{m n}-\lambda_{00}\right) \frac{1}{Z_{01}^{\text {ex3 }}}}
$$

where $\lambda_{m n}:=\lambda_{m n}(k f)$ are the prolate spheroidal eigenvalues (see p.11 in [10]).

Remark 8 By construction, we have $Z_{00}^{\mathrm{DtN1} 13 \mathrm{~d}}=Z_{00}^{\mathrm{DtN2}, 3 \mathrm{~d}}=Z_{00}^{\mathrm{ex3}}$ and $Z_{01}^{\mathrm{DtN2}, 3 \mathrm{~d}}=$ $Z_{01}^{\text {ex3 }}$. Furthermore, when $e \rightarrow 0$ i.e. the prolate spheroid becomes a sphere, the three-dimensional approximate DtN specific impedances given by Eqs. (41)(42) are identical to the ones obtained in the case of spherical-shaped radiators [18].

The next proposition states the asymptotic behavior, as $k a \rightarrow 0$, of the threedimensional approximate specific impedance $Z_{m n}^{\mathrm{DtN} 2,3 \mathrm{~d}}$. This result is an immediate consequence of substituting the asymptotic behavior of the exact specific impedance given by Eq. (40) into Eq. (42) and from using the following property of the prolate spheroidal eigenvalues (see p.11 in [10]):

$$
\lambda_{m n}(0)=n(n+1) \quad \text { and } \quad m \leq n
$$

Proposition 3.1 The asymptotic behavior, as $k a \rightarrow 0$, of the three-dimensional approximate DtN2 specific impedance for the $m n^{\text {th }}$ prolate spheroidal mode is given by:

$$
Z_{m n}^{\mathrm{DtN} 2,3 \mathrm{~d}} \sim-2 \frac{n(n+1)-2}{(n(n+1)+2)^{2}}(k a)^{2}-\mathrm{i} \frac{2 k a}{n(n+1)+2} ; \quad n \geq 2 \quad(m \leq n)
$$


Remark 9 First, observe that the asymptotic behavior, as $k a \rightarrow 0$, of $Z_{m n}^{\mathrm{DtN1} 1,3 \mathrm{~d}}$ is identical to the behavior of the exact specific impedance $Z_{00}^{\text {ex3 }}$ given by Eq. (40). In addition, for $(m, n)=(0,0)$ and $(0,1)$, the asymptotic behavior of $Z_{m n}^{\mathrm{DtN2,3d}}$ is identical to the behavior of the exact specific impedance $Z_{m n}^{\mathrm{ex} 3}$. Second, for $n \geq 2$, the asymptotic behavior of $Z_{m n}^{\mathrm{DtN} 2,3 \mathrm{~d}}$ is identical to the case of a sphere (see Eq. (92), p. 32 in [18]) (independent of the eccentricity e), as well as to the approximate specific impedance corresponding to BGT2 condition when applied on a prolate spheroid radiator (see Eq. (69), p. 3646 in [24]). Last, the asymptotic behavior given by Eq. (40) and Eq. (44) indicates that for higher modes, the real part of the exact impedance tends to zero, as $k a \rightarrow 0$, faster than the DtN2 specific impedance. This result suggests that the three-dimensional DtN2 boundary condition may not be appropriate for single higher prolate spheroidal modes.

Next, we investigate numerically the performance of the designed approximate local DtN boundary conditions when computing three-dimensional single radiating higher order modes $(n \geq 2)$. Recall that by construction DtN2 is exact for the first two modes $((m, n)=(0,0)$ and $(0,1))$, and therefore outperforms BGT2 when expressed in prolate spheroidal coordinates (see Eq. (60) p. 3645 in Reference [24]). We have performed several experiments to assess the effect of the wavenumber and the slenderness of the boundary on the performance of the second-order DtN boundary condition DtN2 given by Eq. (5). All the obtained results are reported in $[5,26]$. These results indicate, as demonstrated analytically, that overall both DtN2 and BGT2 absorbing boundary conditions perform poorly when computing higher modes. This conclusion is clearly illustrated by the numerical results depicted in Figs. (9)-(11). The results represent the relative errors when computing two single higher prolate spheroidal modes $((m, n)=(0,2)$ and $(1,1))$, for two wavenumber values: $k a=0.1$ (see Figs. (9), (11)) and $k a=1$ (see Figs. (10), (12)). These results have been obtained for six eccentricity values $e=0.1,0.2$ corresponding to a prolate-spheroid boundary "close" to a sphere, $e=0.4,0.6$ corresponding to a "regular" prolate-spheroid boundary, and $e=0.8,0.9$ corresponding to a "very" elongated prolate-spheroid boundary. Note that we have reported the obtained results for the relative errors as a function of $\varphi$ since the approximate BGT2 specific impedance depends on $\varphi \in[0, \pi)$ (see Eq. (69) in [24]). Observe that for $k a=0.1$ (see Figs. (9), (11)) the relative error is about $20 \%$ for most eccentricity values and observation angle $\varphi$. The situation is slightly better for $k a=1$ (see Figs. (10), (12)), yet the level of accuracy remains very high for practitioners. Note that the level of accuracy deteriorates significantly when computing single higher prolate spheroidal modes as expected and observed in $[5,26]$. 
$e=0.1$

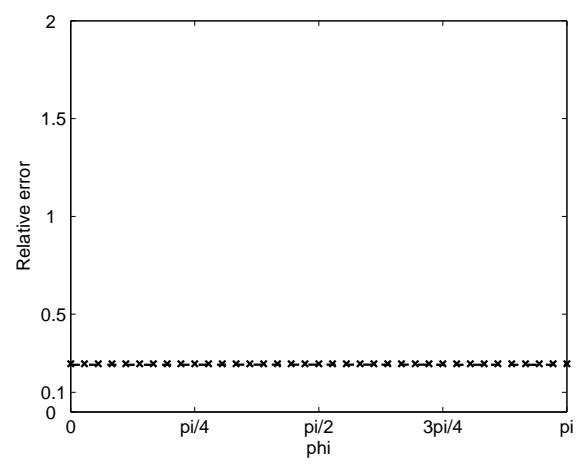

$e=0.4$

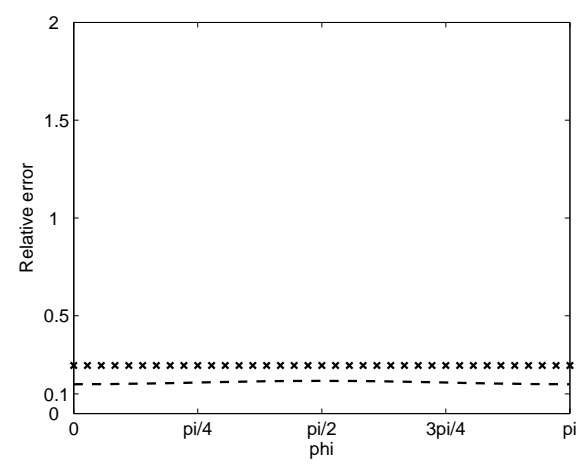

$e=0.8$

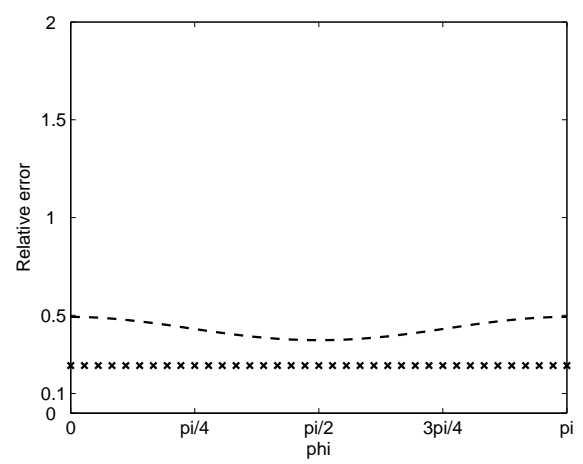

$e=0.2$

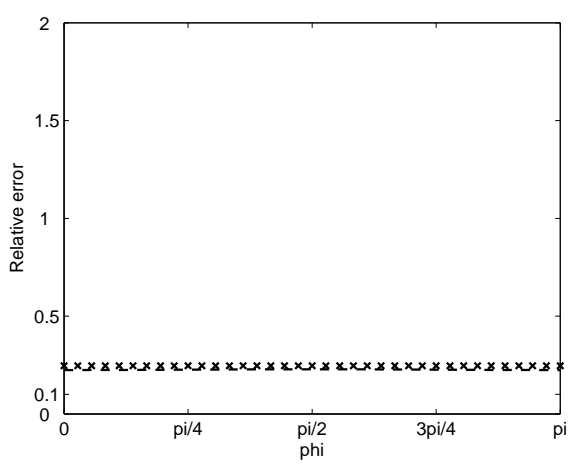

$e=0.6$

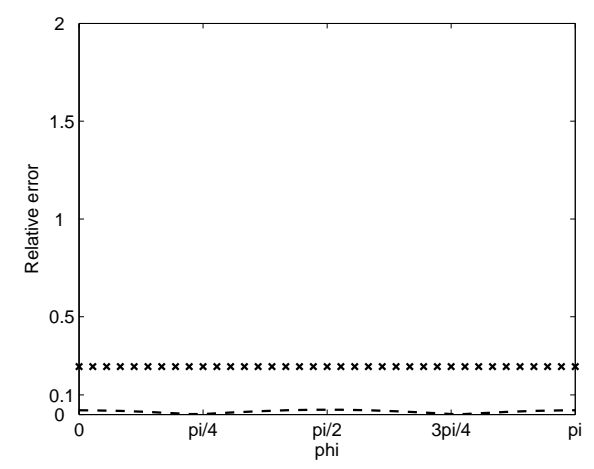

$e=0.9$

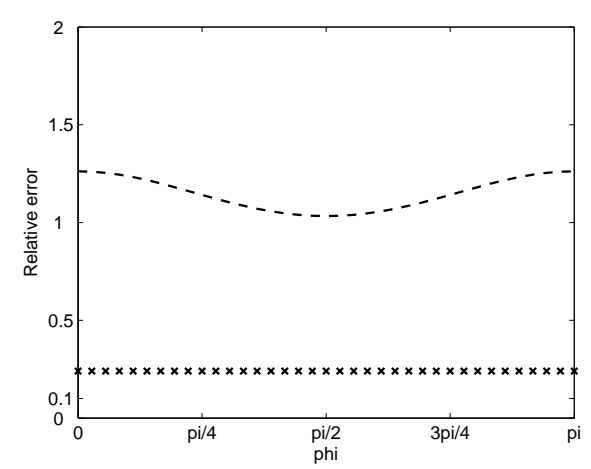

Fig. 9. Relative error of the specific impedance for DtN2 (crossed), BGT2 (dashed) when computing the prolate spheroidal mode $(m, n)=(0,2)$ for $k a=0.1$. 
$e=0.1$

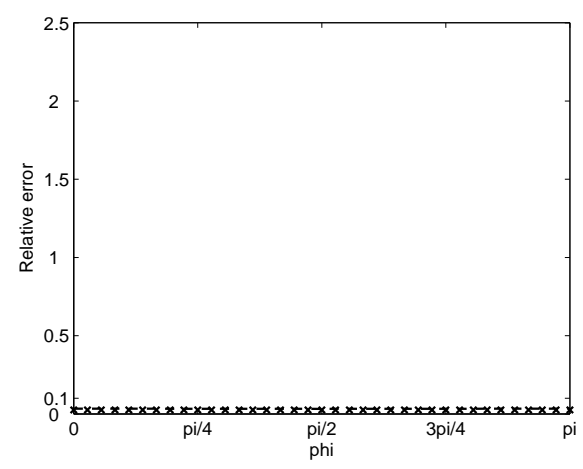

$e=0.4$

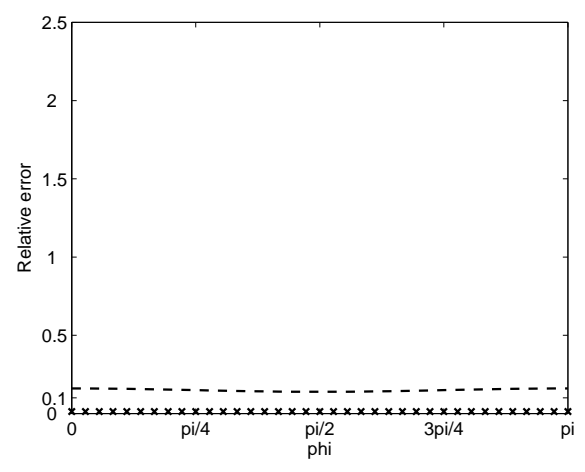

$e=0.8$

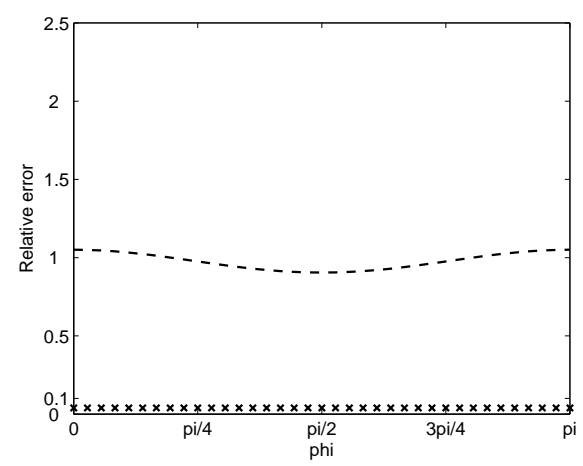

$e=0.2$

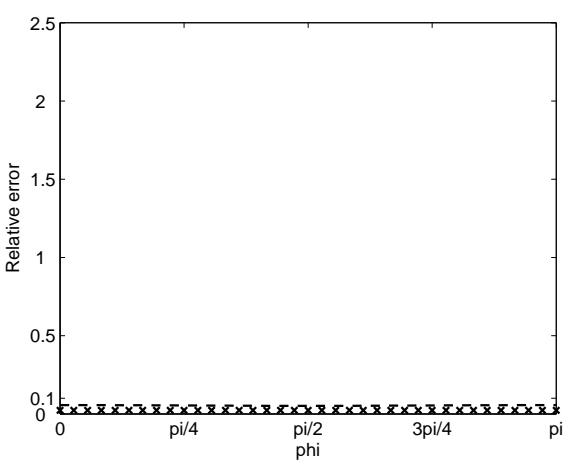

$e=0.6$

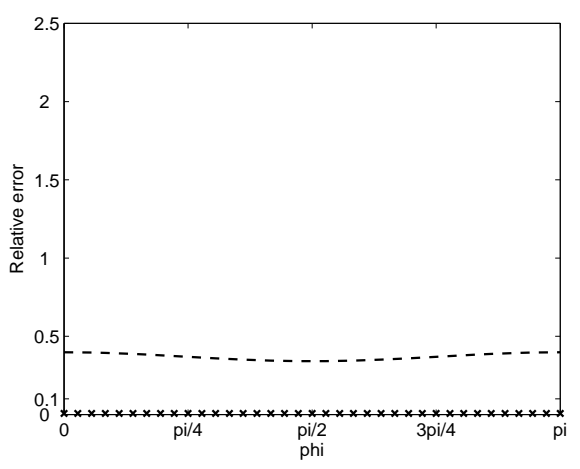

$e=0.9$

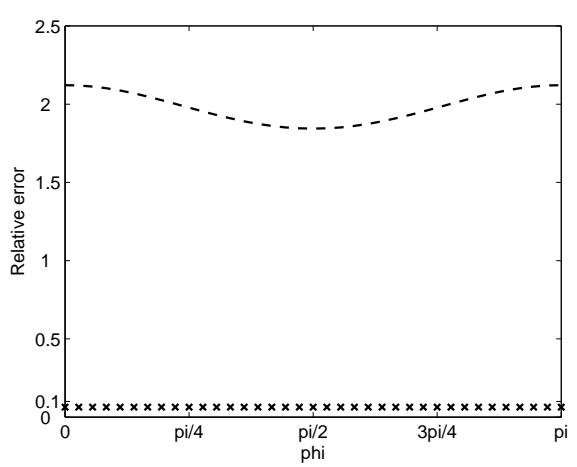

Fig. 10. Relative error of the specific impedance for DtN2 (crossed), BGT2 (dashed) when computing the prolate spheroidal mode $(m, n)=(0,2)$ for $k a=1$. 

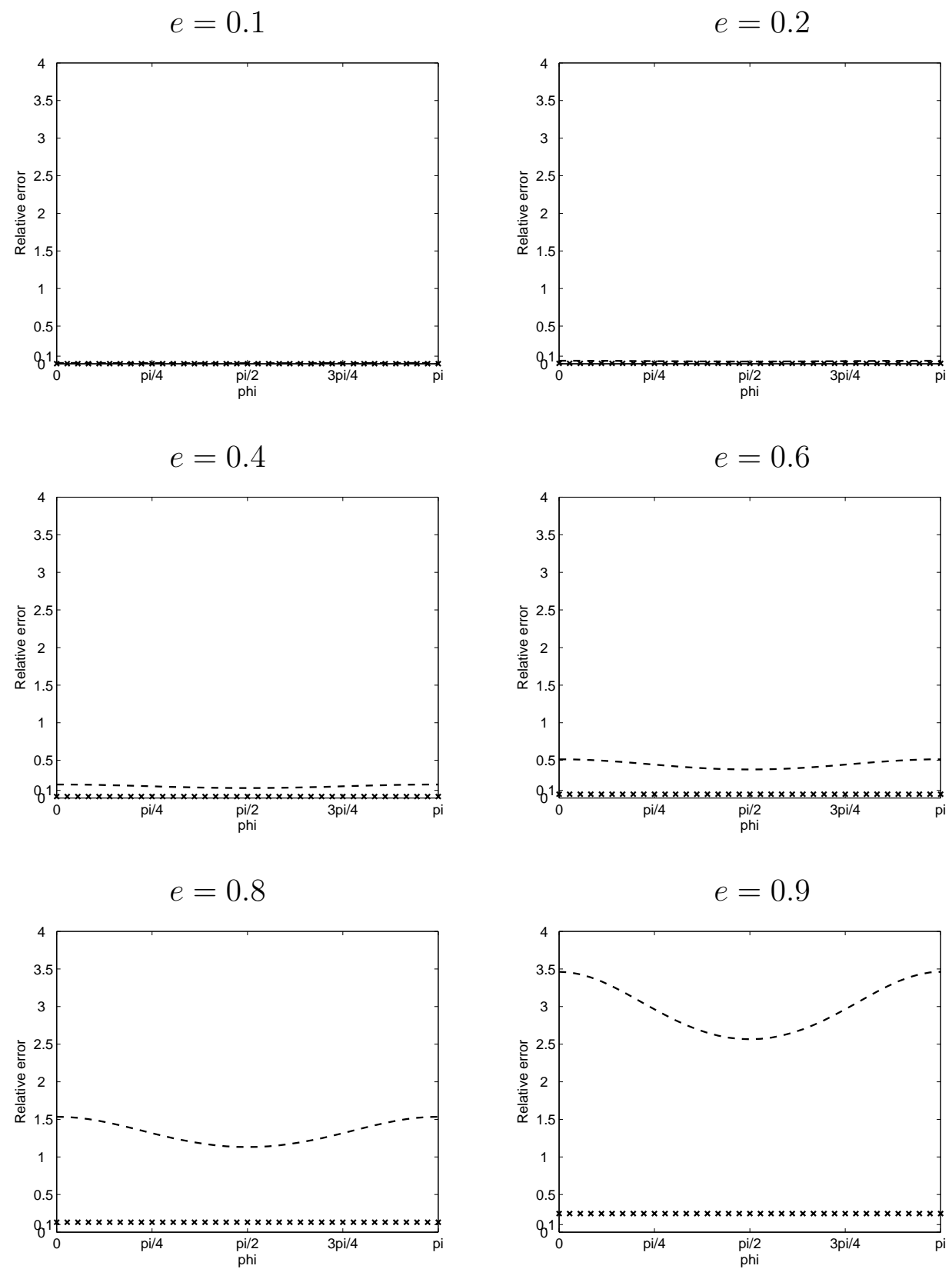

Fig. 11. Relative error of the specific impedance for DtN2 (crossed), BGT2 (dashed) when computing the prolate spheroidal mode $(m, n)=(1,1)$ for $k a=0.1$. 
$e=0.1$

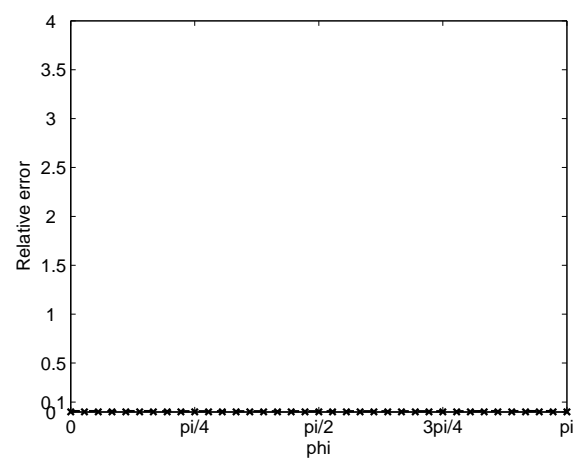

$e=0.4$

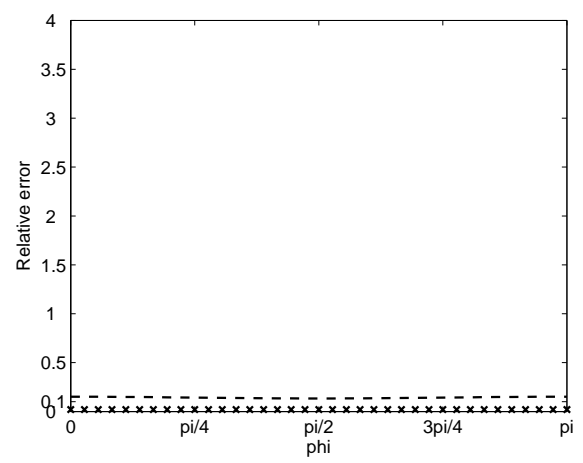

$e=0.8$

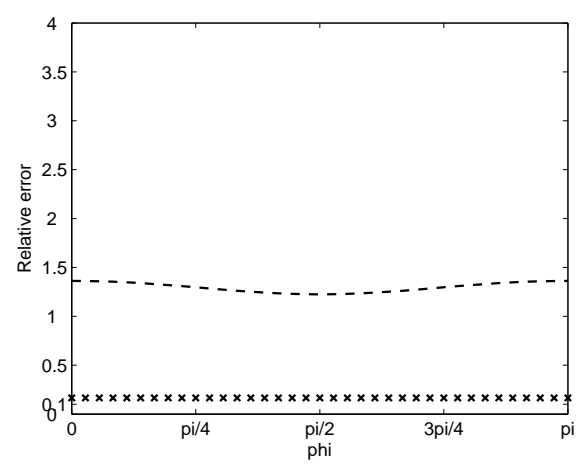

$e=0.2$

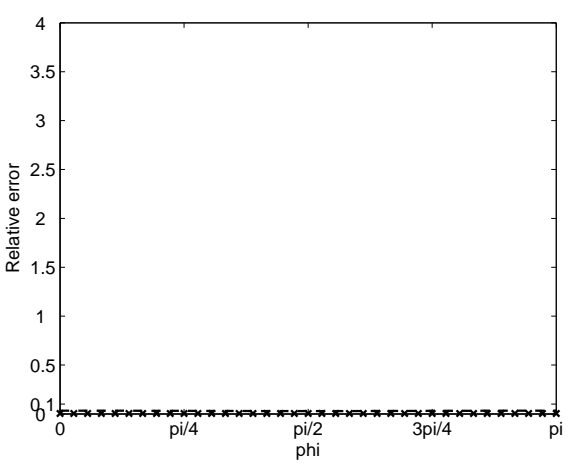

$e=0.6$

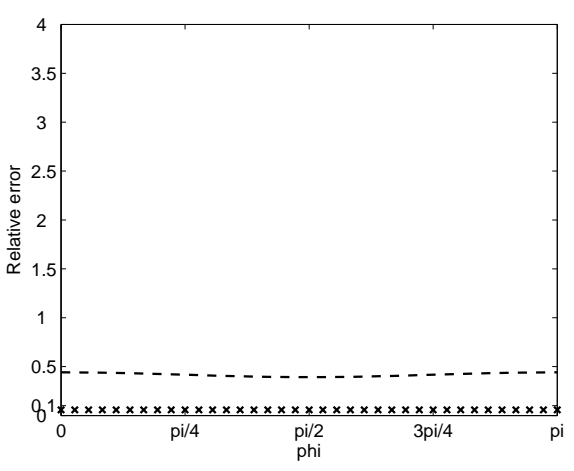

$e=0.9$

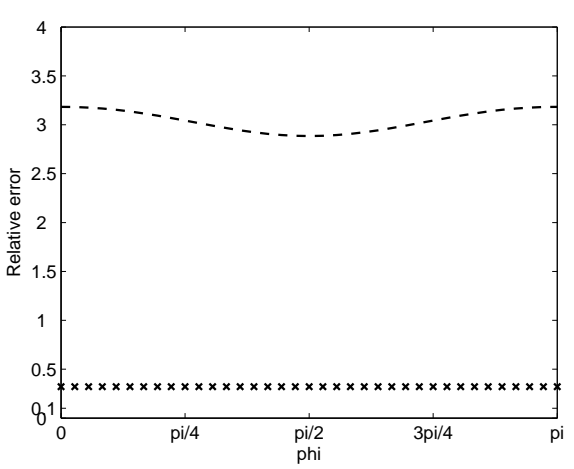

Fig. 12. Relative error of the specific impedance for DtN2 (crossed), BGT2 (dashed) when computing the prolate spheroidal mode $(m, n)=(1,1)$ for $k a=1$. 


\subsection{Performance analysis for three-dimensional scattering problems}

This paragraph is devoted to the analysis of the effect of the wavenumber and eccentricity values on the performance of the approximate local DtN absorbing boundary conditions given by Eqs. (31)-(32) when applied for solving threedimensional acoustic scattering problems by sound-soft obstacles. We assume the scatterer to be a rigid prolate spheroid and the incident field $u^{\text {inc }}$ to be a plane wave with incident angle $\varphi_{0}$ given, in prolate spheroid coordinates, by:

$$
u^{\mathrm{inc}}=\mathrm{e}^{\mathrm{i} k f \cosh \xi\left(\cos \varphi \cos \varphi_{0}+\tanh \xi \sin \varphi \sin \varphi_{0} \cos \theta\right)}
$$

Therefore, the acoustic scattered field $u^{\text {scat }}$ can be represented by the following series [27]:

$$
u^{\text {scat }}=-2 \sum_{m=0}^{\infty} \sum_{n=m}^{\infty}\left(2-\delta_{0 m}\right) A_{m n} i^{n} \mathrm{R}_{m n}^{(3)}(k f, \cosh \xi) \mathrm{S}_{m n}(k f, \cos \varphi) \cos m \theta
$$

where $\delta_{0 m}$ is the Kronecker delta symbol, the coefficients $A_{m n}$ is given by:

$$
A_{m n}=\frac{\mathrm{R}_{m n}^{(1)}\left(e k a, e^{-1}\right) \mathrm{S}_{m n}\left(e k a, \cos \varphi_{0}\right)}{N_{m n} \mathrm{R}_{m n}^{(3)}\left(e k a, e^{-1}\right)}
$$

and $N_{m n}$ is the normalization factor of the angular spheroidal wave functions $[10]$.

Consequently, the exact specific impedance $Z^{\mathrm{ex} 3}$ on the surface of a prolate spheroid at $\xi=\xi_{0}$ is given by (see Eq. (85), p. 3649 in [24]):

$$
Z^{\mathrm{ex} 3}=-\frac{\mathrm{i} \sqrt{1-e^{2}} k a u^{\mathrm{inc}}}{\left.\frac{\partial}{\partial \xi}\left(u^{\mathrm{scat}}\right)\right|_{\xi=\xi_{0}}}
$$

and its asymptotic behavior, as $k a \rightarrow 0$, is given by (see Eq. (91), p. 3655 in $[24])$ :

$$
Z^{\mathrm{ex} 3} \sim Z_{00}^{\mathrm{ex} 3} \sim(k a)^{2}-\mathrm{i} k a
$$

where $Z_{00}^{\text {ex3 }}$ is given by Eq. (39).

The next lemma states the expressions of the approximate specific impedances on the boundary $\xi=\xi_{0}$ of a prolate-spheroid sound-soft scatterer. This lemma results, as demonstrated in [26], from a straightforward substitution into Eq. (7) of $u=-u^{\text {inc }}$ (sound-soft scatterer), and from using the approximate local DtN boundary conditions given by Eqs. (31)-(32) to evaluate $\frac{\partial u}{\partial \xi}=-\frac{\partial u^{\text {inc }}}{\partial \xi}$ at $\xi=\xi_{0}$. 
Lemma 10 The three-dimensional approximate specific impedance $Z^{\mathrm{DtN1}, 3 \mathrm{~d}}$, for a sound-soft scattering problem, corresponding to DtN1 absorbing boundary condition is given by:

$$
Z^{\mathrm{DtN} 1,3 \mathrm{~d}}=Z_{00}^{\mathrm{ex} 3}
$$

The three-dimensional approximate specific impedance $Z^{\mathrm{DtN2}, 3 \mathrm{~d}}$, for a soundsoft scattering problem, corresponding to DtN2 absorbing boundary condition is given by:

$$
Z^{\mathrm{DtN} 2,3 \mathrm{~d}}=\frac{\lambda_{01}-\lambda_{00}}{\left(\frac{\lambda_{01}}{Z_{00}^{\mathrm{ex} 3}}-\frac{\lambda_{00}}{Z_{01}^{\mathrm{ex} 3}}\right)\left(-2 i \alpha k a-(k a)^{2} \delta-(e k a)^{2} \cos \varphi\right)}
$$

where

$$
\begin{aligned}
& \alpha=\cos \varphi \cos \varphi_{0}+\sqrt{1-e^{2}} \sin \varphi \sin \varphi_{0} \cos \theta \\
& \delta=\left(\frac{\partial \alpha}{\partial \varphi}\right)^{2}+\frac{1}{\sin ^{2} \varphi}\left(\frac{\partial \alpha}{\partial \theta}\right)^{2}
\end{aligned}
$$

Remark 11 Note that when $e \rightarrow 0$, i.e. the prolate-spheroid becomes a sphere, the approximate DtN specific impedances given by Eqs. (50) and (51) are identical to the ones obtained in the case of a spherical-shaped scatterer $[18]$.

The next proposition states the asymptotic behavior, as $k a \rightarrow 0$, of the threedimensional approximate specific impedance $Z^{\mathrm{DtN2}, 3 \mathrm{~d}}$. This result is an immediate consequence of substituting into Eq. (51) the asymptotic behavior of the exact specific impedance given by Eq. (40) along with using the property of the prolate spheroidal eigenvalues given by Eq. (43).

Proposition 3.2 The asymptotic behavior, as $k a \rightarrow 0$, of the three-dimensional approximate DtN2 specific impedance for the sound-soft scattering problem is given by:

$$
Z^{\mathrm{DtN} 2,3 \mathrm{~d}} \sim(1-\alpha)(k a)^{2}-\mathrm{i} k a
$$

where $\alpha$ is given by Eq. (52).

Remark 12 It follows from Eqs. (49)-(50) that the asymptotic behavior, as $k a \rightarrow 0$, of $Z^{\text {DtN1,3d }}$ is identical to the behavior of the exact specific impedance $Z^{\mathrm{ex} 3}$. Moreover, Eq. (53) indicates that the asymptotic behavior of $Z^{\mathrm{DtN2}, 3 \mathrm{~d}}$ depends on the eccentricity as well as on the observation angles $(\varphi, \theta)$. This dependence is comparable to the asymptotic behavior of the BGT2 approximate specific impedance (see Eq. (93), p. 3657 in [24]). Note that when $e \rightarrow 0$, i.e. the prolate-spheroid becomes a sphere, the approximate DtN specific impedances given by Eqs. (50) and (51) are identical to the case of spheres (see Eq. (140) p. 42 in [18]). 
Next we investigate numerically the effect of the wavenumber and the slenderness of the boundary on the performance of the approximate local DtN boundary condition given by Eq. (32) when applied for solving sound-soft scattering problems by prolate-spheroid obstacles. We have performed such investigations in the OSRC context [21], and have compared the results to the ones obtained with BGT2 condition when expressed in prolate spheroidal coordinates (see Figs. (22) to (33) in [24]). All the results are reported in $[5,26]$. For illustration purpose, we present the results for only two values of the wavenumeber, $k a=0.1$ and 1 , corresponding to three different values of the incidence angle $\varphi_{0}=0, \frac{\pi}{4}$, and $\frac{\pi}{2}$. These results have been obtained for six eccentricity values $e=0.1,0.2$ corresponding to a prolate spheroid "close" to a sphere, $e=0.4,0.6$ corresponding to a "regular" prolate spheroid boundary, and $e=0.8,0.9$ corresponding to a "very" elongated prolate spheroid. Note that since all the approximate specific impedances depend on the observation angles $\varphi \in[0, \pi)$ and $\theta \in[0,2 \pi)$, we have reported in Figs. (13)-(18) the relative errors as a function of $(\varphi, \theta)$. The following two observations are noteworthy:

i. Unlike the radiating problem, DtN2 absorbing boundary condition retains an excellent level of accuracy when solving acoustic problems for low wavenumbers (the relative error is below $2 \%$ for all eccentricity values). In addition, the results depicted in Figs. (13)-(18) clearly demonstrate that such good performance is not sensitive to the value of the eccentricity $e$. These results suggest in particular that DtN2 absorbing boundary condition given by Eq. (32) is appropriate for elongated boundaries.

ii. DtN2 absorbing boundary condition clearly outperforms the second-order BGT2 absorbing boundary condition especially for high eccentricity values. Indeed, there is a significant loss of accuracy for the BGT2 boundary condition when $e \geq 0.6$ (the relative error is larger than $40 \%$ ). This demonstrates that DtN2 absorbing boundary condition extends the range of satisfactory performance to all eccentricity values in the low frequency regime. 


$$
e=0.1
$$

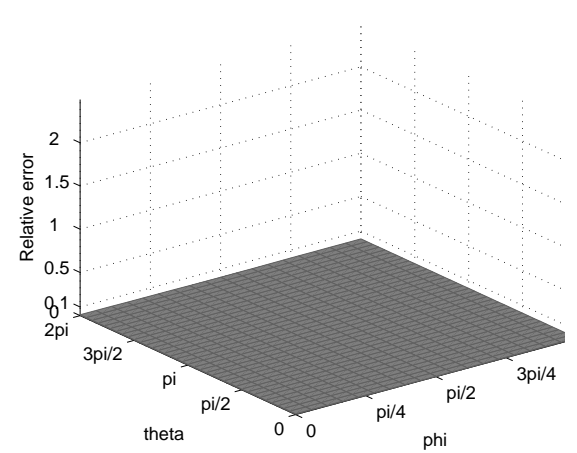

$$
e=0.4
$$

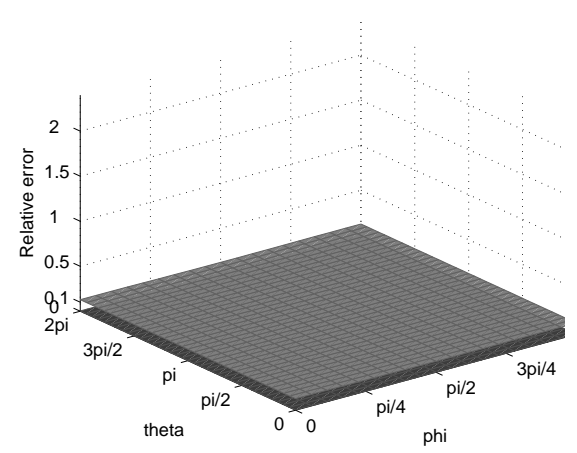

$$
e=0.8
$$

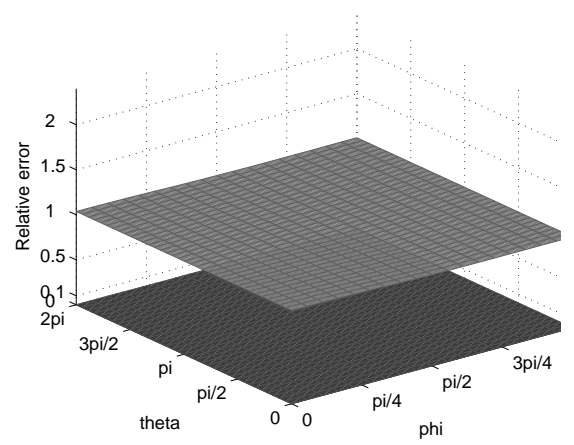

$e=0.2$

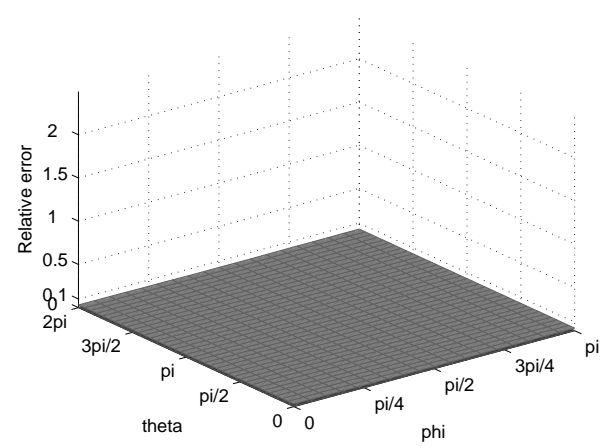

$$
e=0.6
$$
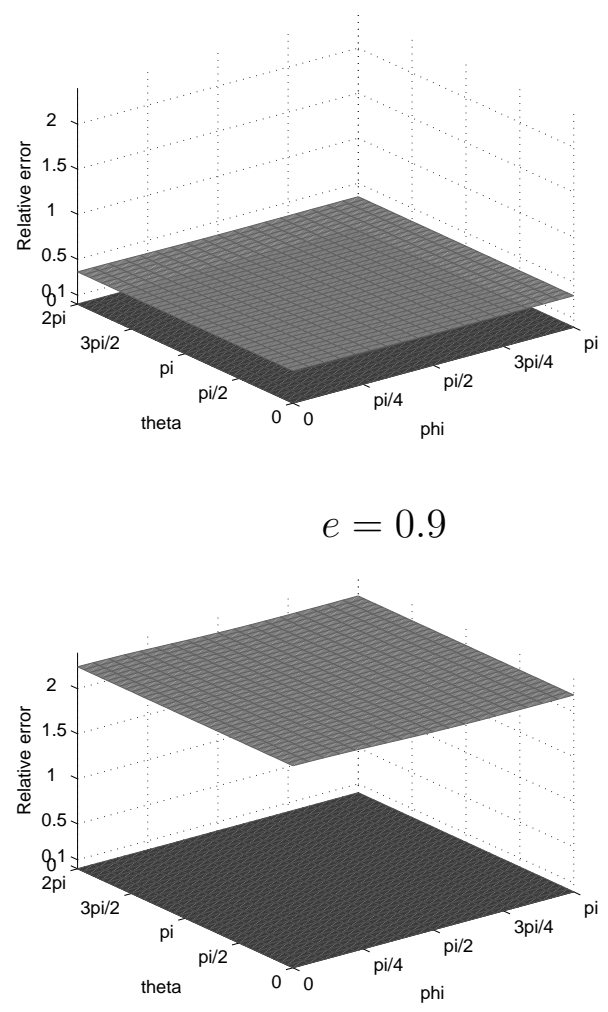

Fig. 13. Relative error of the specific impedance corresponding to DtN2 (black) and BGT2 (dark grey) when solving three-dimensional sound-soft scattering problem with $k a=0.1$ and incident angle $\varphi_{0}=0$. 


$$
e=0.1
$$

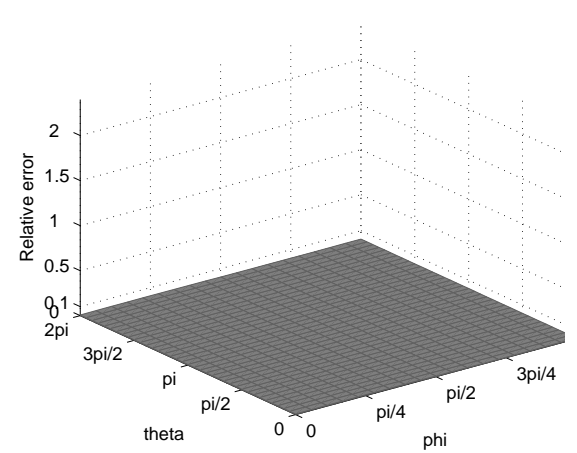

$$
e=0.4
$$

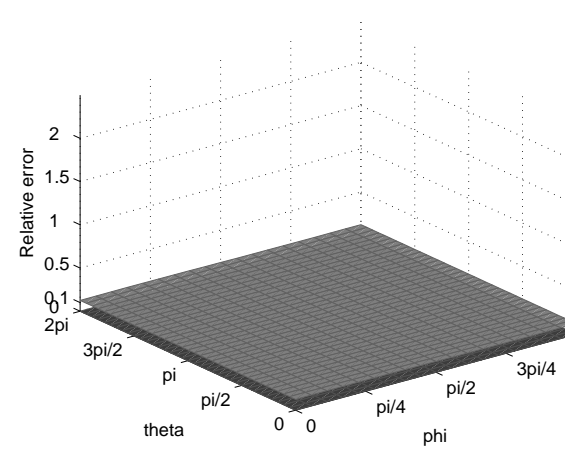

$$
e=0.8
$$

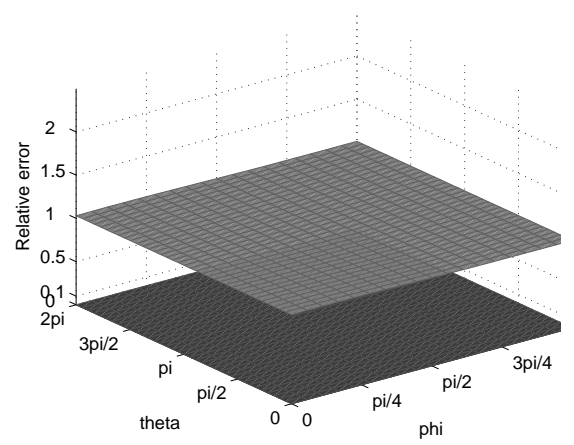

$e=0.2$

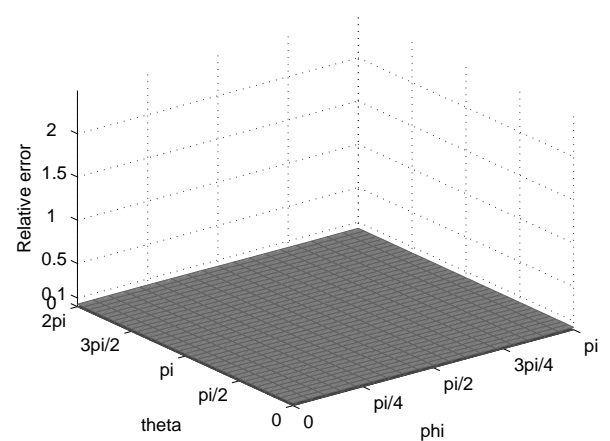

$$
e=0.6
$$
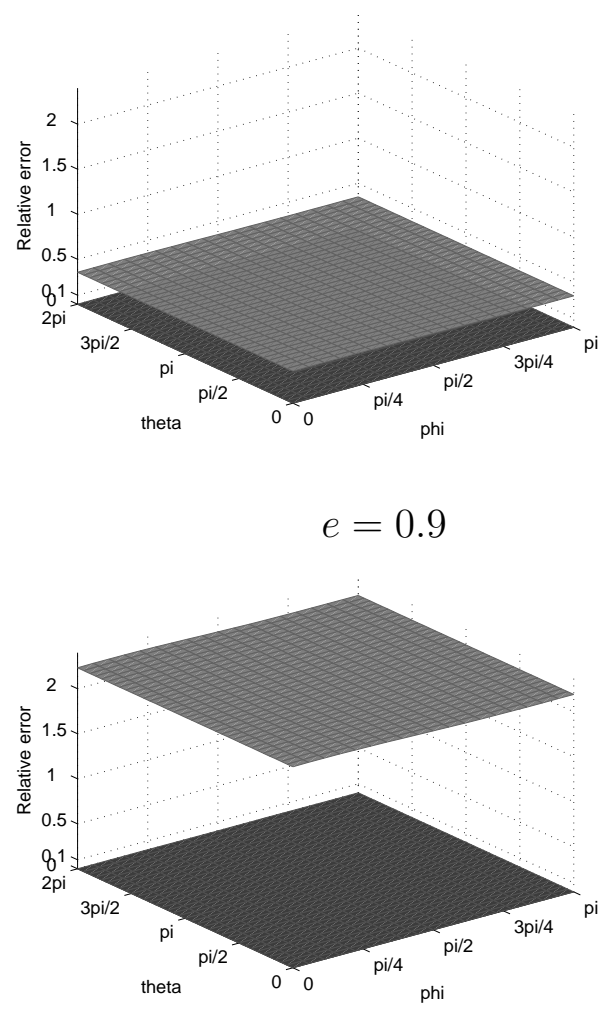

Fig. 14. Relative error of the specific impedance corresponding to DtN2 (black) and BGT2 (dark grey) when solving three-dimensional sound-soft scattering problem with $k a=0.1$ and incident angle $\varphi_{0}=\frac{\pi}{4}$. 


$$
e=0.1
$$

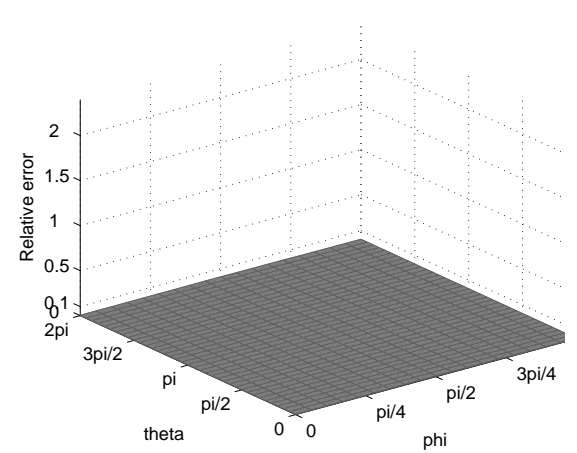

$$
e=0.4
$$

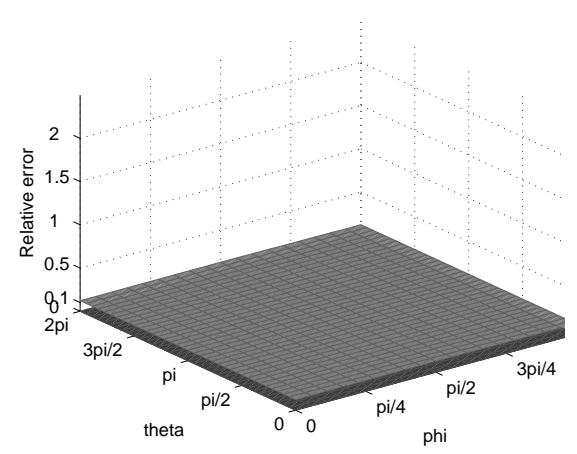

$$
e=0.8
$$

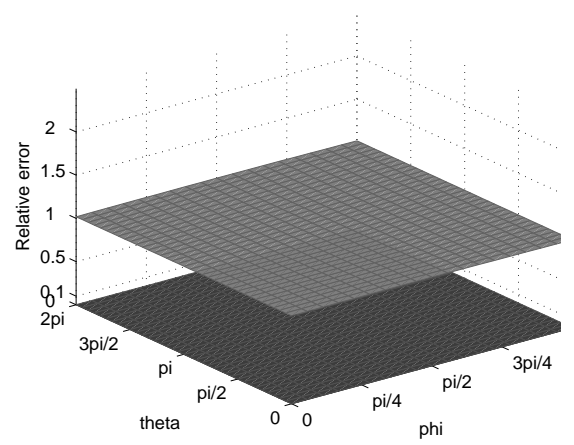

$e=0.2$

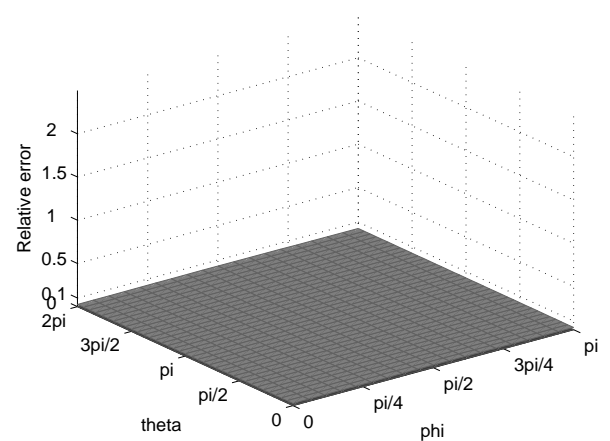

$$
e=0.6
$$
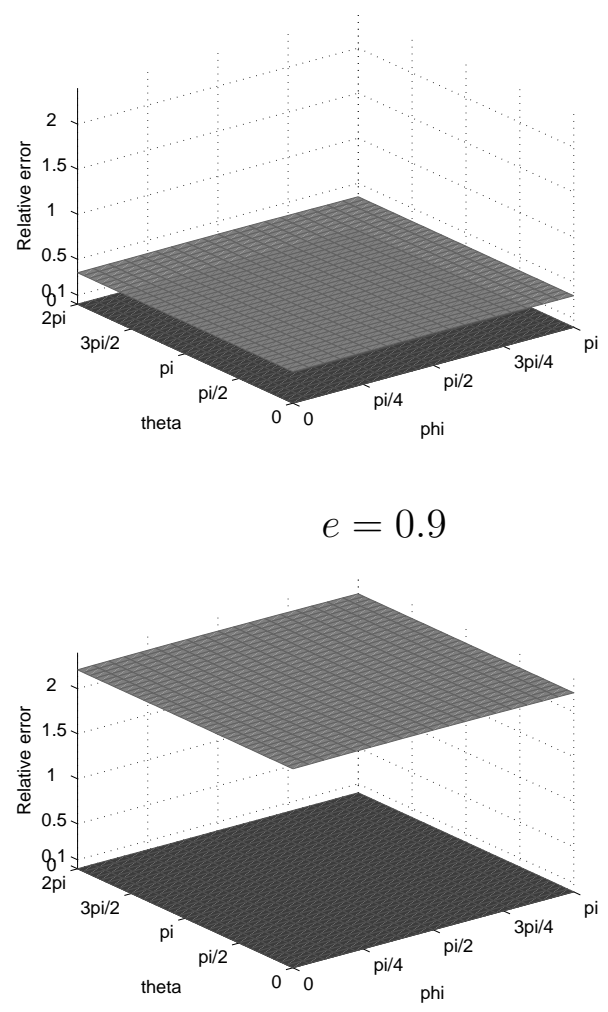

Fig. 15. Relative error of the specific impedance corresponding to DtN2 (black) and BGT2 (dark grey) when solving three-dimensional sound-soft scattering problem with $k a=0.1$ and incident angle $\varphi_{0}=\frac{\pi}{2}$. 


$$
e=0.1
$$

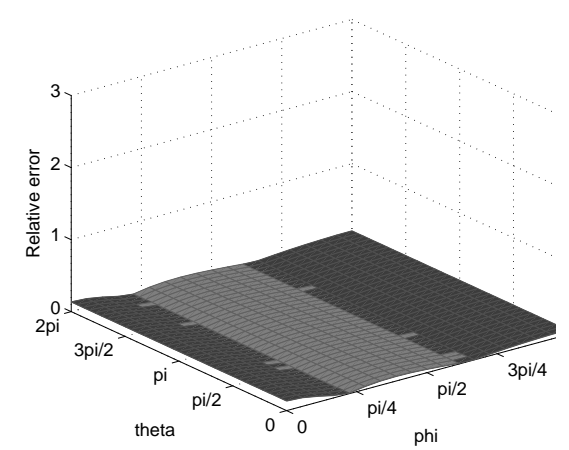

$$
e=0.4
$$

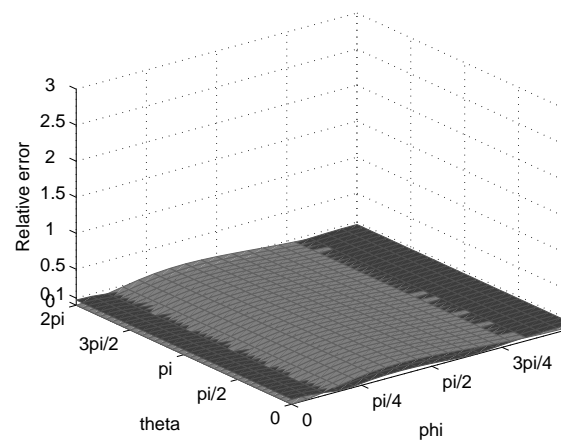

$$
e=0.8
$$

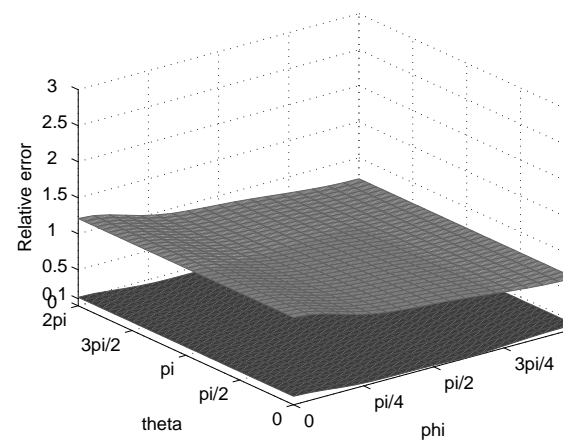

$e=0.2$

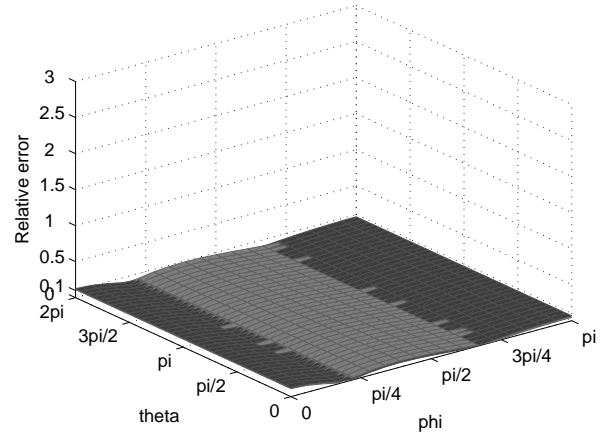

$e=0.6$

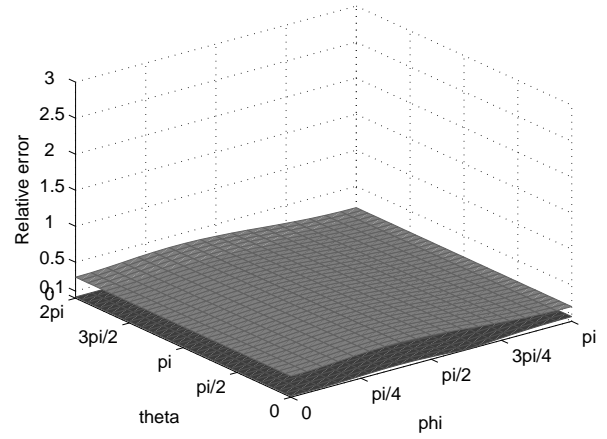

$$
e=0.9
$$

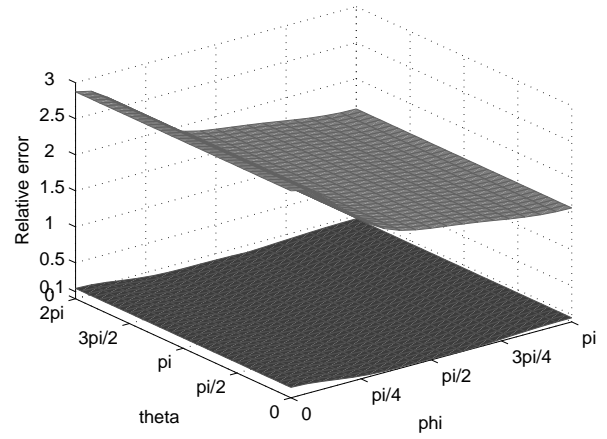

Fig. 16. Relative error of the specific impedance corresponding to DtN2 (black) and BGT2 (dark grey) when solving three-dimensional sound-soft scattering problem with $k a=1$ and incident angle $\varphi_{0}=0$. 


$$
e=0.1
$$

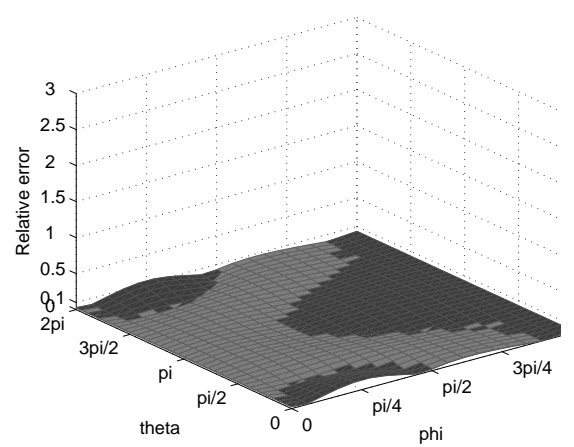

$$
e=0.4
$$

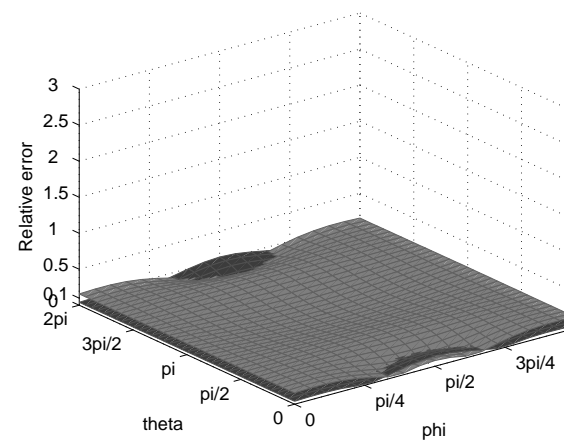

$$
e=0.8
$$

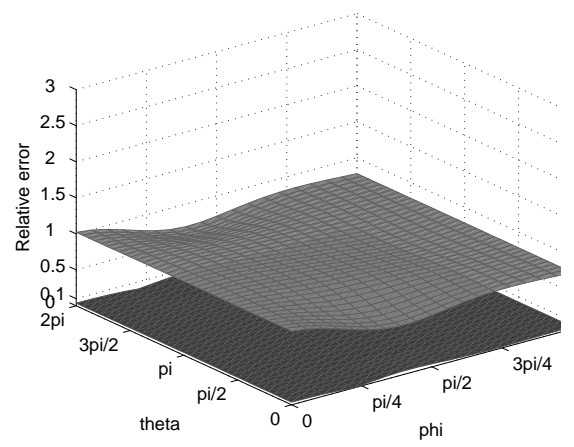

$e=0.2$

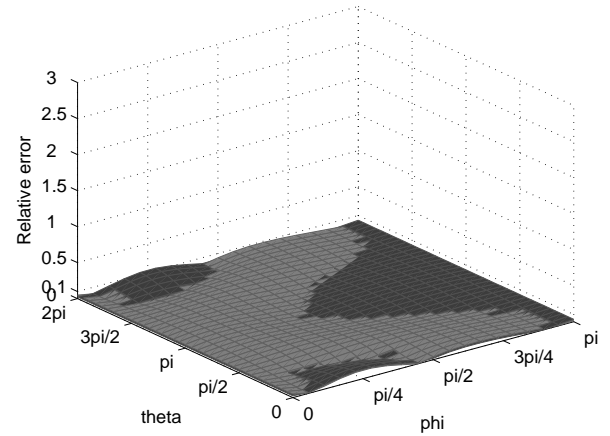

$e=0.6$

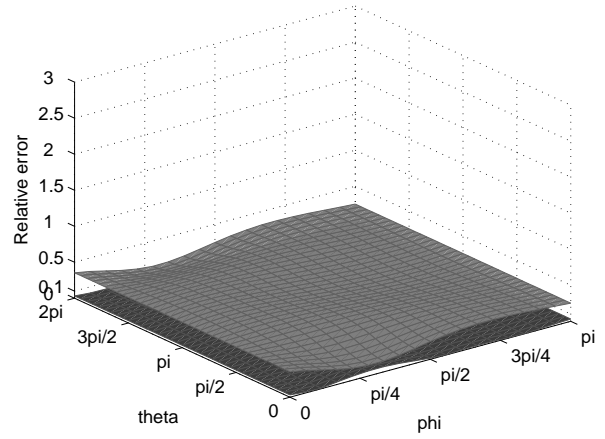

$$
e=0.9
$$

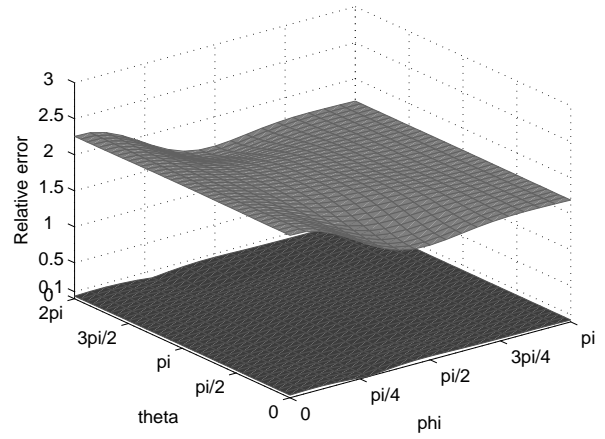

Fig. 17. Relative error of the specific impedance corresponding to DtN2 (black) and BGT2 (dark grey) when solving three-dimensional sound-soft scattering problem with $k a=1$ and incident angle $\varphi_{0}=\frac{\pi}{4}$. 


$$
e=0.1
$$

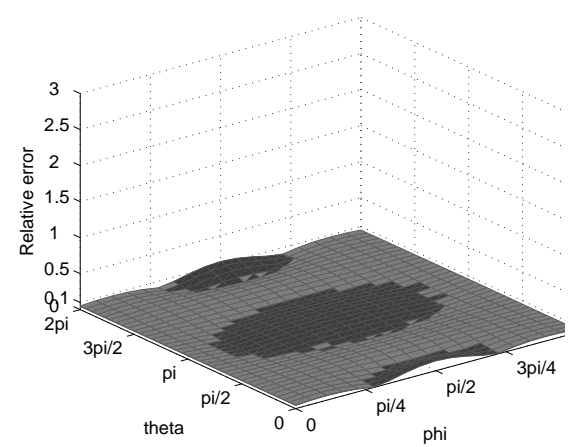

$$
e=0.4
$$

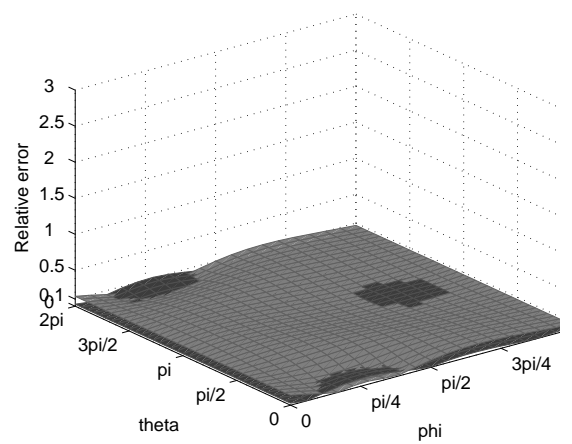

$$
e=0.8
$$

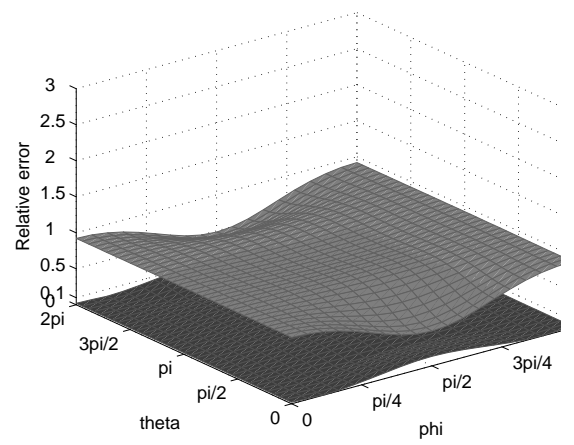

$e=0.2$

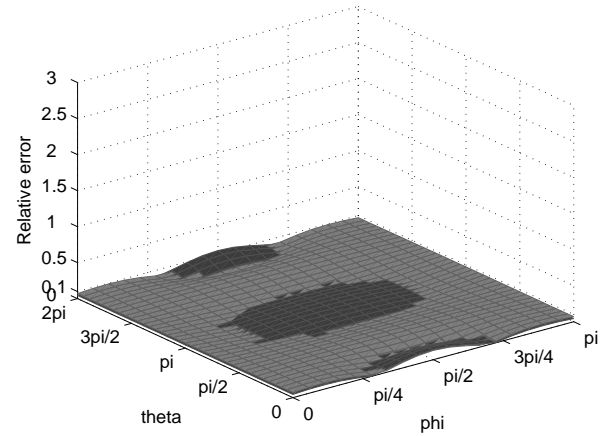

$$
e=0.6
$$
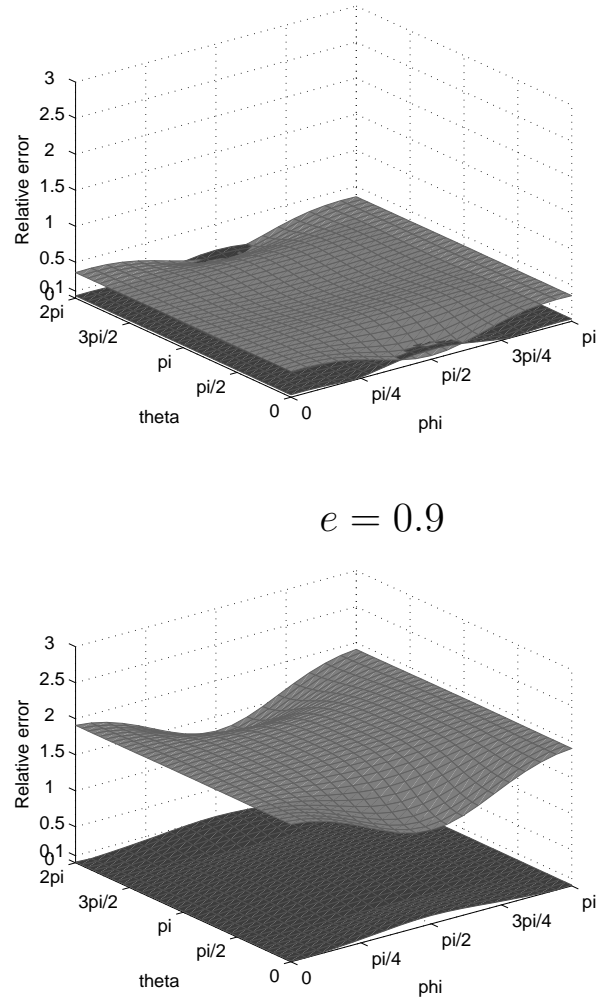

Fig. 18. Relative error of the specific impedance corresponding to DtN2 (black) and BGT2 (dark grey) when solving three-dimensional sound-soft scattering problem with $k a=1$ and incident angle $\varphi_{0}=\frac{\pi}{2}$. 


\section{Conclusion}

We have designed a new class of approximate local ABCs to be applied on elliptical-shaped exterior boundaries when solving acoustic scattering problems by elongated obstacles. These conditions are exact for the first radiation modes, they are easy to implement and to parallelize, and they preserve the local structure of the computational finite element scheme. The analysis reveals that in the case of the radiator, DtN2 boundary condition, by construction, outperforms the BGT2 condition when computing the first single modes, while for higher single modes both conditions perform poorly. Note that for higher modes and high eccentricity values, DtN2 delivers a better level of accuracy, yet this level is still not acceptable for most applications. The situation for scattering problems is very different. BGT2 boundary condition performs poorly for eccentricity values $e \geq 0.6$ (in $3 \mathrm{D}$ case for example, the relative error is larger than 40\%), while the DtN2 boundary condition delivers an excellent level of accuracy in the low frequency regime for all eccentricity values (in $3 \mathrm{D}$ case for example, the relative error is smaller than $2 \%$ ). This investigation demonstrates that DtN2 absorbing boundary condition extends the range of satisfactory performance to all eccentricity values in the low frequency regime.

\section{Acknowledgements}

The authors acknowledge the support by INRIA/CSUN Associate Team Program. Any opinions, findings, conclusions or recommendations expressed in this material are those of the authors and do not necessarily reflect the views of INRIA or CSUN.

\section{References}

[1] M. Abramovitz, I. Stegun, Handbook of Mathematical Functions with Formulas, Graphs and Mathematical Tables, Dover Publications, New York, 1972

[2] X. Antoine, Fast approximate computation of a time-harmonic scattered field using the on-surface radiation condition method, IMA J. Appl. Math., 66(1):83110,2001

[3] X. Antoine, M. Darbas, and Y. Y. Lu, An improved surface radiation condition for high-frequency acoustic scattering problems, Comput. Methods Appl. Mech. Engrg., 195, 4060-4074, 2006

[4] H. Barucq, R. Djellouli, A. Saint-Guirons, Construction and performance analysis of local DtN absorbing boundary conditions for exterior Helmholtz 
problems. Part I : Elliptical shaped boundaries, NRIA Research Report, No. 6394 (2007). Available online at: http://hal.inria.fr/inria-00180471/fr/

[5] H. Barucq, R. Djellouli, A. Saint-Guirons, Construction and performance analysis of local DtN absorbing boundary conditions for exterior Helmholtz problems. Part II : Prolate spheroid boundaries,INRIA Research Report, No.6395 (2007). Available online at: http://hal.inria.fr/inria-00180475/fr/

[6] A. Bayliss, M. Gunzburger, and E. Turkel, Boundary conditions for the numerical solution of elliptic equations in exterior regions, SIAM J. Appl. Math., 42 (2), pp. 430-451, 1982

[7] J.J. Bowman, T.B.A. Senior, P.L.E. Uslenghi, Electromagnetic and acoustic scattering by simple shapes, North-Holland Publishing company, Amsterdam, 1969

[8] D. C. Calvo, M. D. Collins, D. K. Dacol, A higher-order On-Surface Radiation Condition derived from an analytic representation of a Dirichlet-to-Neumann map, IEEE Transaction on Antennas and Propagation, 51 (7), pp. 1607-1614, 2003

[9] D. C. Calvo and A. Wilde-Angle, On-Surface Radiation Condition applied to scattering by spheroids, Journal of the Acoustical Society of America 116 (3), pp. 1549-1558, 2004

[10] C. Flammer, Spheroidal Functions, Standford University Press, Standford, CA, 1957

[11] T. L. Geers, Doubly asymptotic approximations for transient motions of submerged structures, J. Acoust. Soc. Am., 64 (5), pp. 1500-1508, 1978

[12] T. L. Geers, Third-order doubly asymptotic approximations for computational acoustics, J. Comput. Acoust., 8 (1), pp. 101-120, 2000

[13] D. Givoli and J. B. Keller, Nonreflecting boundary conditions for elastic waves, Wave Motion, 12(3), pp. 261-279, 1990

[14] D. Givoli, Exact representations on artificial interfaces and applications in mechanics, AMR, 52(11), pp. 333-349, 1999

[15] M. J. Grote, J. B. Keller, On nonreflecting boundary conditions, J. Comput. Phys., 122(2), pp. 231-243, 1995

[16] I. Harari, Computational methods for Problems of Acoustics with Particular Reference to Exterior Domains, PhD thesis, Stanford university, May 1991

[17] I. Harari and T. J. R. Hughes, Analysis of continuous formulations underlying the computation of time-harmonic acoustics in exterior domains, Comput. Methods Appl. Mech. Engrg., 97(1), pp. 103-124, 1992

[18] I. Harari, R. Djellouli, Analytical study of the effect of wave number on the performance of local absorbing boundary conditions for acoustic scattering, Applied Numerical Mathematics, 50, pp. 15-47, 2004 
[19] R. Kechroud, X. Antoine, and A. Soulaimani, Numerical Accuracy of a padétype non-reflecting boundary condition for the finite element solution of acoustic scattering problems at high-frequency, International Journal for Numerical Methods in Engineering 64 (10), pp. 1275-1302, 2005

[20] J. B. Keller, D. Givoli, Exact nonreflecting boundary conditions, J. Comput. Phys., 82 (1), pp. 172-192,1989

[21] G. A. Kriegsmann, A. Taflove, and K. R. Umashankar, A new formulation of electromagnetic wave scattering using an on-surface radiation boundarycondition approach, IEEE Trans. Antennas and Propagation 35(2), pp. $153-161,1987$

[22] M. Medvinsky, E. Turkel, and U. Hetmaniuk, Local absorbing boundary conditions for elliptical shaped boundaries, J. Comput. Phys., 227 (18), pp. 8254-8267, 2008

[23] J. Meixner, F.W.Schafle, Matieusche funktionen and spharoidfunktionen, Springer Verlag, Berlin, 1954

[24] R.C. Reiner, R. Djellouli, and I. Harari, The performance of local absorbing boundary conditions for acoustic scattering from elliptical shapes, Comput. Methods Appl. Mech. Engrg, 195, 3622-3665, 2006

[25] R.C. Reiner and R. Djellouli, Improvement of the performance of the BGT2 condition for low frequency acoustic scattering problems, Journal of Wave Motion, 43, pp. 406-424, 2006

[26] A. Saint-Guirons, Construction et analyse de conditions aux limites absorbantes pour des problèmes de propagation d'ondes, Ph.D. thesis, Univerisité de Pau et des Pays de l'Adour, France (In Preparation)

[27] T. B. A. Senior, Scalar diffraction by a prolate spheroid at low frequencies, Canad. J. Phys. 38 (7), pp. 1632-1641, 1960

[28] J.A. Stratton, Electromagnetic theory, McGraw-Hill, New York, 1941

[29] E. Turkel, Iterative methods for the exterior Helmholtz equation including absorbing boundary conditions, In: Computational Methods for Acoustics Problems, F. Magoulès (ed.), Saxe-Coburg Publications, 2008 